\title{
Nested contour dynamics models for axisymmetric vortex rings and vortex wakes
}

\author{
Clara O'Farrell and John O. Dabiri \\ ${ }^{1}$ Control and Dynamical Systems, California Institute of Technology, Pasadena CA 91125, USA \\ ${ }^{2}$ Graduate Aeronautical Laboratories and Bioengineering, California Institute of Technology, \\ Pasadena CA 91125 , USA
}

(Received 26 May 2013; revised 9 March 2014; accepted 5 April 2014;

first published online 1 May 2014)

Inviscid models for vortex rings and dipoles are constructed using nested patches of vorticity. These models constitute more realistic approximations to experimental vortex rings and dipoles than the single-contour models of Norbury and Pierrehumbert, and nested contour dynamics algorithms allow their simulation with low computational cost. In two dimensions, nested-contour models for the analytical Lamb dipole are constructed. In the axisymmetric case, a family of models for vortex rings generated by a piston-cylinder apparatus at different stroke ratios is constructed from experimental data. The perturbation response of this family is considered by the introduction of a small region of vorticity at the rear of the vortex, which mimics the addition of circulation to a growing vortex ring by a feeding shear layer. Model vortex rings are found to either accept the additional circulation or shed vorticity into a tail, depending on the perturbation size. A change in the behaviour of the model vortex rings is identified at a stroke ratio of three, when it is found that the maximum relative perturbation size vortex rings can accept becomes approximately constant. We hypothesise that this change in response is related to pinch-off, and that pinch-off might be understood and predicted based on the perturbation responses of model vortex rings. In particular, we suggest that a perturbation response-based framework can be useful in understanding vortex formation in biological flows.

Key words: biological flows, propulsion, vortex dynamics

\section{Introduction}

Vortex rings are a recurring feature in a wide class of biological, atmospheric and engineering flows (Turner 1960; Shariff \& Leonard 1992; Lugt 1995; Gharib et al. 2006). In particular, vortex rings are often found in the wakes of swimming and flying animals (Rayner 1979; Dickinson et al. 2000; Linden \& Turner 2004; Dabiri \& Gharib 2005a). Given their ubiquity in nature and technology, vortex rings have been the subject of numerous studies. Most commonly, vortex rings are generated in experimental studies using a 'piston-cylinder' arrangernent. In these experiments, a fluid column of length $L$ is ejected through the circular aperture or nozzle at the end of a hollow cylinder of diameter $D$ by a piston moving inside the cylinder with

† Email address for correspondence: jodabiri@ caltech.edu 
a prescribed velocity $U_{p}$. Gharib, Rambod \& Shariff (1998) showed that the growth of vortex rings during fluid ejection cannot continue indefinitely, but rather there is a physical limit to their size. Beyond this limit, which occurred a stroke ratio of $L / D \approx 4$, Gharib et al. (1998) found that vortex rings stopped accepting vorticity during fluid ejection and additional vorticity flux formed a wake of Kelvin-Helmoltztype vortices instead. They termed the disconnection of the velocity and vorticity fields of the vortex ring from its feeding shear layer 'vortex pinch-off'. Krueger \& Gharib (2003) have demonstrated that the pinch-off process has dynamical significance, as the efficiency of momentum transport in pulsed jets is optimised when the size of the vortex rings generated by the jet is maximised. In addition, there is evidence that some natural systems make use of this phenomenon for efficient fluid transport (Linden \& Turner 2004; Dabiri \& Gharib 2005a; Gharib et al. 2006; Dabiri et al. 2010).

Gharib et al. (1998) attributed the occurrence of pinch-off to an energy-based argument due to Kelvin and Benjamin (Kelvin 1880; Benjamin 1976), which states that a vortex ring is a steady solution of the incompressible Euler equations only when it has maximum energy with respect to rearrangements of the vorticity density that preserve the same total impulse. However, in practice it is difficult to show that a particular vortex ring with a realistic vorticity distribution maximises the total energy with respect to alternative configurations, as outlined by the Kelvin-Benjamin principle. As a result, Gharib et al. (1998) relied on a combination of modelling and empirical results to show that, at a vortex formation time $\left(\hat{T}=\bar{U}_{p} t / D\right.$, where $\bar{U}_{p}$ is the mean piston speed) of approximately 4 , the piston-cylinder vortex generator apparatus cannot meet the impulse-normalised energy requirements necessary to generate an isolated vortex ring.

Since the original model of Gharib et al. (1998) relied on empirical input, a number of models explaining and attempting to predict pinch-off were developed subsequently, including those of Mohseni \& Gharib (1998), Shusser \& Gharib (2000), Linden \& Turner (2001), Kaplanski \& Rudi (2005), Fukumoto \& Kaplanski (2008) and Gao \& Yu (2010). Despite this wealth of models, no single one has emerged as the preferred means of predicting pinch-off. This is due, in part, to existing models not being easily applicable to all biological and engineering flows. Most existing models rely on quantifying the circulation, impulse, energy or velocity of the growing vortex ring, as well as of the shear layer feeding it. Therefore, these models prove difficult to apply when the flux of circulation and energy into the vortex ring, or the velocity of the shear layer, cannot be easily quantified. In these cases, which include most examples of swimming and flying animals, the utility of existing models is limited. In these complex biological flows, criteria for identifying and predicting pinch-off based on the properties of the vortex rings in the wake alone are desirable. In this study, we consider the perturbation response of models for these vortex rings, in order to explore the link between the behaviour of the wake vortices and pinch-off.

Notably, both the argument of Gharib et al. (1998) and the models of Mohseni \& Gharib (1998), Shusser \& Gharib (2000), Linden \& Turner (2001) and Gao \& Yu (2010) make use of a family of vortex rings introduced by Norbury (1973) as a model for axisymmetric vortex rings. The Norbury family consists of steadily translating axisymmetric vortex rings with finite core size, ranging from classical thin-cored vortices to Hill's spherical vortex. In all members of the family, the vorticity density $(\omega / r$, where $r$ is the radial coordinate) is constant inside the core. While vortex formation and pinch-off is an unsteady process, a quasi-steady approximation has been employed successfully in the past to model the dynamics of the growing experimentally generated vortex rings using the steady Norbury vortices. In the 
studies of Gharib et al. (1998), Mohseni \& Gharib (1998), Shusser \& Gharib (2000), Linden \& Turner (2001) and Gao \& Yu (2010), members of the Norbury family of vortices serve as low-order models of experimentally generated vortex rings of different core sizes.

In a previous study (O'Farrell \& Dabiri 2012), we considered the phenomenon of pinch-off by studying the perturbation response of the different members of the Norbury family using contour dynamics methods. We found a change in the response of the perturbed vortices as we considered vortices of increasing core size, which was analogous to pinch-off. Given that these vortices have been used to model vortex rings at different stages in their growth, this change in response suggests that the perturbation response of models for isolated vortex rings could be a useful tool in understanding the pinch-off of vortex rings in real flows. Since pinch-off was not expected to occur in two dimensions (Afanasyev 2006; Pedrizzetti 2010), this conjecture was supported by the fact that no such change in response was observed when we considered perturbations to a two-dimensional analogue to the Norbury family of vortex rings, namely the Pierrehumbert family of vortex dipoles (Pierrehumbert 1980).

However, the vortex rings and dipoles considered in O'Farrell \& Dabiri (2012) consisted of single patches inside which the vorticity was constant (in the twodimensional case) or a linear function of the distance from the axis of symmetry (in the axisymmetric case). In contrast, experimentally generated vortex rings are characterised by a Gaussian distribution of vorticity (Weigand \& Gharib 1997) and the vorticity in experimentally generated dipoles is well-approximated by Bessel functions (Flór \& van Heijst 1994; Trieling et al. 2010). Therefore, there is room for improvement of our understanding of the relationship between the perturbation response of models for isolated vortex rings and dipoles, and the pinch-off phenomenon observed in laboratory flows and in the field, by considering more realistic models for the vortices. In two dimensions, more realistic vortex models with continuous distributions of vorticity have been previously studied by Boyd \& Ma (1990), Kizner \& Khvoles (2004), Khvoles, Berson \& Kizner (2005) and Albrecht, Elcrat \& Miller (2011), and others; whereas Kaplanski \& Rudi (2005) and Fukumoto \& Kaplanski (2008) have considered similar models for vortex rings. Unlike the inviscid solutions of Norbury (1973) and Pierrehumbert (1980), these models were viscous and studying their perturbation response required the use of viscous flow solvers.

More realistic models for both vortex rings and dipoles can be constructed, while preserving the computational advantages of the contour dynamics formulation, by considering multiple nested patches of vorticity. This arrangement enables the approximation of more realistic continuous distributions of vorticity by piecewiseconstant or piecewise-linear distributions, in two-dimensional and axisymmetric flows, respectively. Thus constructed, the models remain inviscid and their evolution can be computed using contour dynamics algorithms.

In two dimensions, vorticity distributions of arbitrary complexity can be approximated in a piecewise-constant fashion by using multiple nested patches of constant vorticity (Zabusky, Hughes \& Roberts 1979). To date, only a handful of studies have employed nested patches to simulate the evolution of vortices with smooth vorticity distributions. Dritschel (1989) simulated the evolution of an elliptic vortex modelled in a similar way to the elliptic vortices with smooth vorticity distributions considered by Mellander, McWilliams \& Zabusky (1987), using eight nested regions of constant vorticity. Similarly, Pullin \& Jacobs (1986) conducted 
four-contour simulations of Corcos-Lin vortex arrays (Corcos \& Lin 1984; Corcos, Sherman \& Lin 1984; Lin \& Corcos 1984) and Jacobs \& Pullin (1989) utilised eight- and 16-contour approximations to study the evolution of a shear layer with a Gaussian vorticity distribution.

In the case of axisymmetric flows, the contributions from multiple regions of linear vorticity distribution may be combined by linear superposition to allow the simulation of systems with multiple patches, using the axisymmetric contour dynamics algorithm of Shariff, Leonard \& Ferziger (2008). In fact, Shariff et al. (2008) mentioned the possibility of using nested contours to approximate arbitrary vorticity distributions by piecewise-linear ones. However, the authors considered only the case of a 'hollow' spherical vortex, which consisted of a region of zero vorticity nested within a spherical region with a linear distribution of vorticity (i.e. Hill's spherical vortex). To date, no studies that make use of nested contours to construct approximations to experimental vortex rings are available that are more realistic than the Norbury family employed by most existing models for vortex pinch-off.

In this study, we used nested-contour dynamics to construct more realistic inviscid models for the vortex rings found in starting jets, and considered the perturbation response of these models. Model vortex rings were constructed from experimental data from a starting jet, and consisted of several nested contours. Since the model vortices were described by several contours, perturbations to the vortices could be easily introduced in the form of deformations to the shape of the contours. Hence, we analysed the response of the model vortex rings to shape perturbations in a manner similar to our previous analysis of the Norbury family (O'Farrell \& Dabiri 2012).

In order to obtain a more realistic model for the perturbations that vortex rings experience during formation, we devised a perturbation scheme that consisted of deforming the rear boundary of the vortex by introducing a small protuberance. This perturbation mimicked the introduction of a small amount of vorticity at the rear of a forming vortex ring by interaction with its feeding shear layer. Using this perturbation scheme, we were able to identify a change in the behaviour of our model vortex rings that was consistent with pinch-off. Similar methods can be applied to the construction of models for vortex rings in biological flows. Study of the response of these models to a wider class of perturbations could enable prediction of the pinch-off phenomenon in more complex biological flows.

The paper is organised as follows. In $\S 2$ we outline the mathematical and numerical framework for computation of the evolution of multiple regions of vorticity using contour dynamics algorithms. A test case for these methods is presented in $\S 3$, where we model the analytical Lamb dipole using nested contours. In $\S 4$ and $\S 5$ we discuss the construction of model vortex rings from experimental data for a starting jet, and we consider their perturbation response in $\$ 6$. Finally, concluding remarks are presented in $\$ 7$.

\section{Mathematical formulation and numerical method}

\subsection{Contour dynamics formulation for multiple contours}

We considered the evolution of axisymmetric vortex rings and two-dimensional vortices constructed from nested patches of vorticity, using contour dynamics methods. Figure 1 shows a schematic of a symmetric vortex dipole or axisymmetric vortex ring constructed from three nested patches of vorticity. The areas of the nested patches are denoted by $A_{1}, A_{2}$ and $A_{3}$, and distances have been normalised by $R$, which represents the ring radius or half the dipole spacing. 


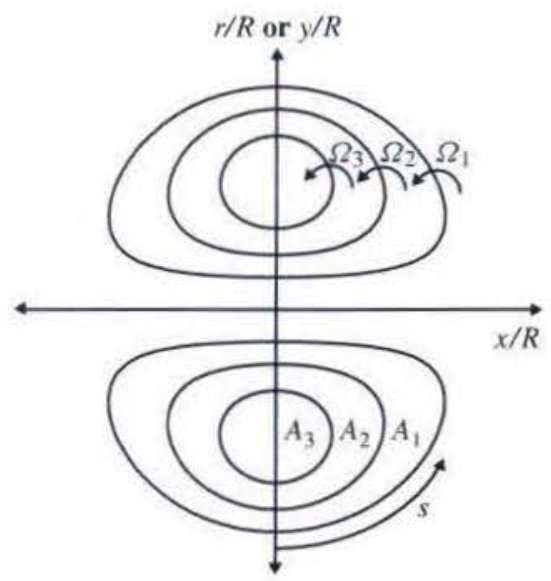

FIGURE 1. The coordinates and parameters defined in the text for nested-contour models for vortex rings and dipoles. Three nested patches $A_{1}, A_{2}$ and $A_{3}$ are shown, and the $\Omega_{i}$ represent the increment in $\Omega$ as each contour is crossed inwards, $s$ is the path coordinate along each contour. The vortices propagate from left to right.

In two dimensions, the velocity induced at a point $z=x+\mathrm{i} y$ in the complex plane by $n$ patches $A_{j}(j=1, \ldots, n)$ of constant vorticity (nested or otherwise) is given by

$$
u_{x}+\mathrm{i} u_{y}=-\frac{1}{4 \pi} \sum_{j=1}^{n} \Omega_{j} \oint_{\partial A_{j}} \frac{z-z^{\prime}}{\bar{z}-\overline{z^{\prime}}} \mathrm{d} z^{\prime},
$$

where $j$ iterates over all contours (Pullin 1981; Dritschel 1988b). Where the contours are nested, $\Omega_{j}$ represents the increment in $\omega$ when crossing the $j$ th contour inwards (see figure 1). When simulating vortex dipoles, we considered only the case where the flow was symmetric about the $x$-axis. In this case, $n$ referred to the number of nested contours comprising one of the symmetric vortex cores, usually taken to be the positive core. The contribution to the velocity from the negatively valued core was derived from symmetry.

Shariff et al. (2008) extended the contour dynamics method to the case of axisymmetric regions with a distribution of vorticity that is linear in the radial coordinate $(\omega=\Omega r$, where $\Omega$ is a constant). The equation for the velocity induced at a point $\boldsymbol{x}$ by $n$ regions $A_{j}$ with a linear distribution of vorticity is given by

$$
\boldsymbol{u}(\boldsymbol{x})=\sum_{j=1}^{n} \Omega_{j} \oint_{\partial A_{j}}\left[\left(x-x^{\prime}\right) G\left(s^{\prime}\right) \cos \theta^{\prime}-r H\left(s^{\prime}\right) \sin \theta^{\prime}\right] \hat{x}+r^{\prime} H\left(s^{\prime}\right) \cos \theta^{\prime} \hat{r} \mathrm{~d} s^{\prime},
$$

where in this case $\Omega_{j}$ represents the jump in $\xi=\omega / r$ when crossing the $j$ th contour inwards, and $H$ and $G$ are given by

$$
\begin{gathered}
G\left(s^{\prime}\right)=\frac{r^{\prime}}{\pi \sqrt{C+B}} K(k), \\
H\left(s^{\prime}\right)=\frac{1}{2 \pi r}\left(\frac{C}{\sqrt{C+B}} K(k)-E(k) \sqrt{C+B}\right), \\
k=\sqrt{\frac{2 B}{C+B}}, \\
C=\left(x-x^{\prime}\right)^{2}+r^{2}+r^{2}, \quad B=2 r r^{\prime} .
\end{gathered}
$$


Here, $\theta^{\prime}\left(s^{\prime}, \alpha\right)$ is the angle of the outward-pointing normal relative to the symmetry axis, and $K(k)$ and $E(k)$ are the complete elliptic integrals of the first and second kind, respectively.

\subsection{Numerical method}

The contour dynamics algorithms described in the preceding section reduced the evolution problem to tracking the motion of a collection of marker points on the boundary of each contour, by numerical integration of (2.1) or (2.2). Numerical integration was carried out using a method similar to that described in O'Farrell \& Dabiri (2012). Contour integration was performed by discretising each contour using linear segments, and evaluating the contribution from segments not adjacent to the field point using Gaussian quadrature. Equation (2.1) is singular as $\left(z^{\prime}-z\right) \rightarrow 0$, but it may be evaluated exactly over linear segments. As a result, the contribution to (2.1) from segments adjacent to the field point was computed analytically. Equation (2.2) is also singular as $\boldsymbol{x}^{\prime} \rightarrow \boldsymbol{x}$, but, unlike in the two-dimensional case, the contributions from segments adjacent to the field point cannot be evaluated exactly due to the presence of elliptic integrals. Hence, we rely on fourth-order log-polynomial approximations to the elliptic integrals of the first and second kind to evaluate the contributions to the velocity from adjacent segments (Shariff et al. 2008).

The solution was marched forward in time using a fourth-order Runge-Kutta scheme. At each time step, additional marker points were inserted where the linear segments stretched beyond $0.016 R$ and removed where segments shrank below $0.004 R$ (Shariff et al. 2008). In the axisymmetric cases, marker points were also inserted to ensure that the length of the segments remained small compared with the radial coordinate of their end points $(\Delta \ell / r<0.15)$, so that the log-polynomial approximations remained valid (Shariff et al. 2008). Following Shariff et al. (2008), the time step was chosen to satisfy $\Delta t=\left(0.05 / \Omega_{0}\right)$, where $\Omega_{0}$ was the vorticity at the centre of the vortex ring core in the axisymmetric case $\left(\Omega_{0}=\sum_{j=1}^{n} \Omega_{j} R\right)$, or the vorticity inside the innermost contour of the positively signed vortex patch in the two-dimensional case $\left(\Omega_{0}=\sum_{j=1}^{n} \Omega_{j}\right)$. The flow invariants (circulation, impulse and energy) were monitored and their change was kept below $0.01 \%$ over one eddy turnover period for the impulse and circulation, and $0.02 \%$ over the same period for the energy.

\subsection{Verification}

In order to verify our implementation of these numerical algorithms, we first considered the evolution of arrangements consisting of only two nested contours. In particular, we considered 'hollow' vortices with a zero-vorticity patch at their centre. Figure 2 shows the evolution of a pair of symmetric annular vortices. The vortices were constructed by removing regions of vortical fluid from one of the steadily translating dipoles described by Pierrehumbert (1980). Following Shariff et al. (2008), the boundaries of the removed regions were chosen to be the interior streamlines of the unperturbed dipole where $\psi= \pm 0.25$. Figure $2(b-e)$ shows the evolution of the hollow vortex pair into a symmetric arrangement of eight vortices connected by thin filaments. The time $t^{*}=U t / R$ was normalised using the speed of the unperturbed dipole $(U)$ and the distance from the symmetry axis of the centre to one of the vortices $(R)$.

The evolution of hollow vortices into patches of vorticity connected by thin sheets was also observed to occur in the axisymmetric case by Shariff et al. (2008). 

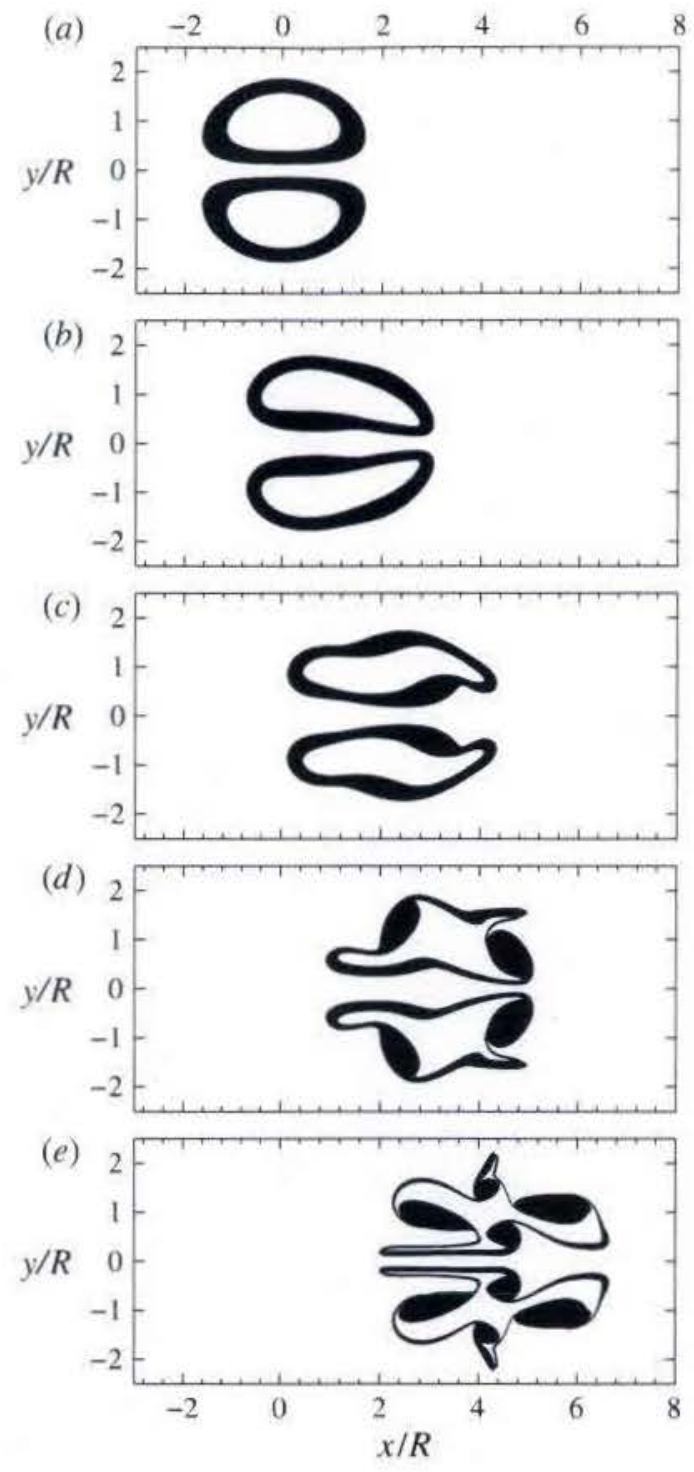

FIGURE 2. The evolution of a pair of annular vortices formed by removing a region of vortical fluid from a Pierrehumbert dipole, at $(a) t^{*}=0$, (b) $t^{*}=7.5,(c) t^{*}=15,(d) t^{*}=$ 22.5 and $(e) t^{*}=30$. The boundaries of the regions removed were the interior streamlines of the unperturbed dipole where $\psi= \pm 0.25$.

Therefore, in order to verify our implementation of the axisymmetric contour dynamics algorithm for multiple contours, we considered the evolution of a hollow spherical vortex constructed by removing a region of vortical fluid from Hill's spherical vortex of radius $2 R$. Following Shariff et al. (2008), the boundary of the region removed was chosen to be an interior streamsurface of the unperturbed Hill's vortex. In our case, the boundary of the removed region was the streamsurface where $\psi=(\Omega / 10) r^{2}\left((2 R)^{2}-r^{2}-x^{2}\right)=0.21$. Figure 3 shows the breakup of the hollow vortex. Once again, the time $t^{*}=U t / R$ was normalised using the speed and radius of the unperturbed Hill's vortex. The results in figure 3 agree qualitatively with the evolution of the vortex described by Shariff et al. (2008). However, since Shariff et al. (2008) did not indicate the streamsurface they selected for their study, a quantitative comparison was not possible. 

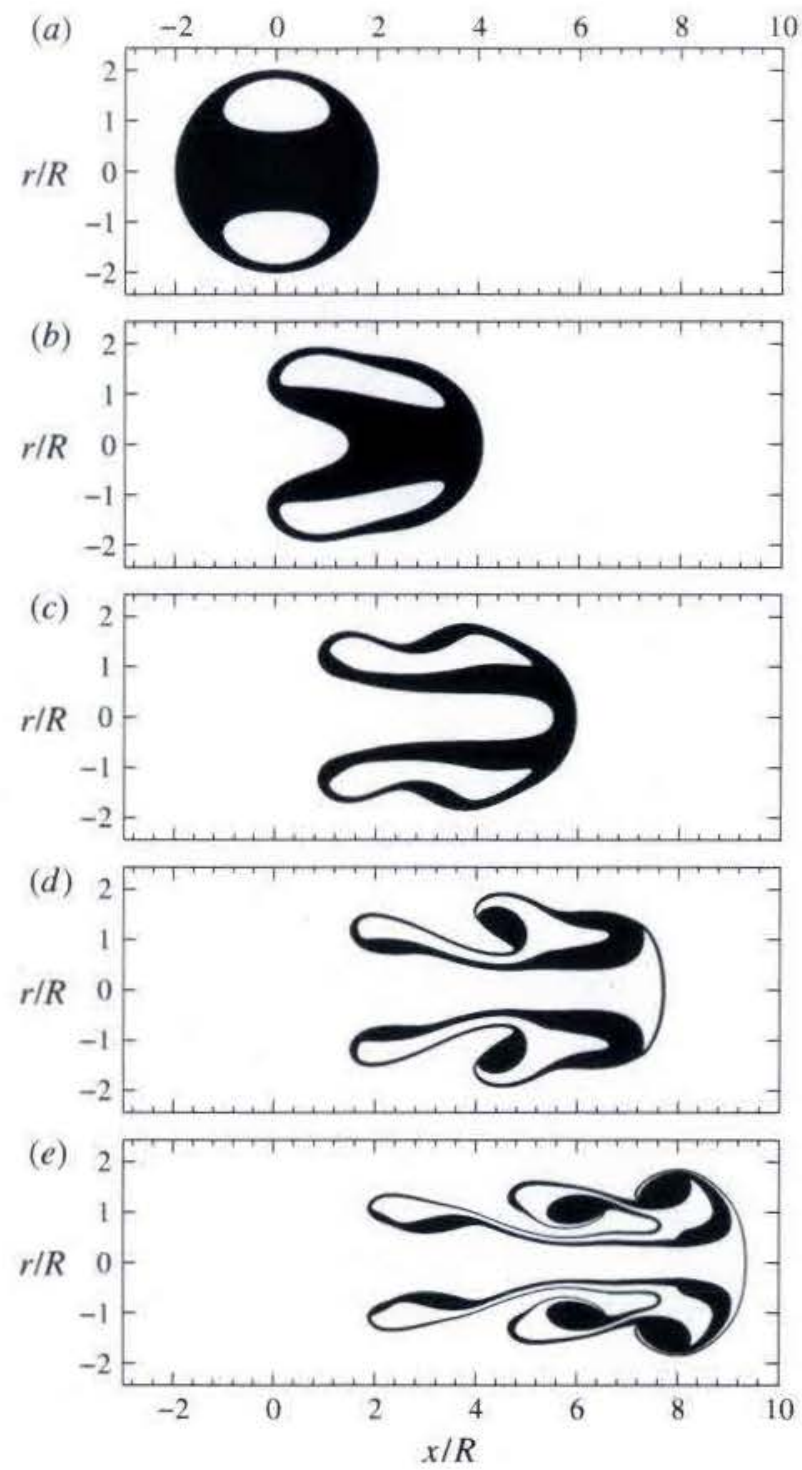

FIGURE 3. The evolution of a hollow spherical vortex formed by removing a region of vortical fluid from Hill's spherical vortex of radius $2 R$, at $(a) t^{*}=0,(b) t^{*}=3.4,(c) t^{*}=$ $6.8,(d) t^{*}=10.2$ and $(e) t^{*}=13.6$. The boundary of the region removed was the interior streamsurface of the unperturbed Hill's vortex where $\psi=(\Omega / 10) r^{2}\left((2 R)^{2}-r^{2}-x^{2}\right)=0.21$.

Having verified our implementation of the contour dynamics algorithms for these simple cases, we proceeded to the construction of a nested-contour model for an exact solution of the incompressible Euler equations in two dimensions: the Lamb dipole.

\section{Example: modelling the Lamb dipole}

The Lamb-Chaplygin dipole is a steadily translating solution to the two-dimensional incompressible Euler equations, which takes the form of a vortex dipole with a circular boundary and a continuous distribution of vorticity. Inside the circular dipole of radius $\rho_{0}$, the vorticity and stream function are linearly related by $\omega=b^{2}(\psi-\lambda)$, where $b$ and $\lambda$ are constants. This general form of the dipole is due to Chaplygin (1903), and was more recently brought to the attention of the scientific community 

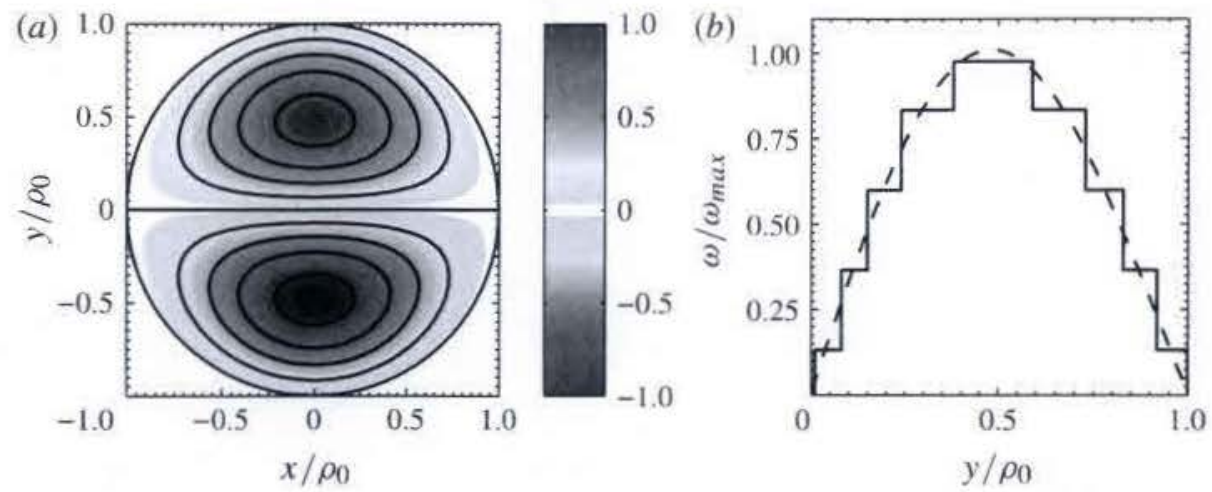

FIGURE 4. The Lamb dipole and a five-contour approximation to the analytical solution. (a) Contours of $\omega / \omega_{\max }$ for the Lamb dipole. The black lines show the locations of five contours used in a piecewise-constant approximation. The dipole propagates from left to right. (b) Analytical (--) and piecewise-constant (-) vorticity distributions along the centreline of the positively valued vortex. The location of the cross-section is shown by a dashed line in $(a)$.

by Meleshko \& van Heijst (1994). Previously, the special case where $\lambda=0$ had been described by Sir Horace Lamb, and is known as the Lamb dipole (Lamb 1895, 1906).

The resulting vorticity field is given by

$$
\omega= \begin{cases}\frac{2 U b}{J_{0}\left(b \rho_{0}\right)} J_{1}(b \rho) \sin \zeta+\lambda b^{2}\left[1-\frac{J_{0}(b \rho)}{J_{0}\left(b \rho_{0}\right)}\right], & \rho \leqslant \rho_{0}, \\ 0, & \rho>\rho_{0},\end{cases}
$$

where $\rho$ and $\zeta$ are the radial and azimuthal coordinates defined from the centre of the circular dipole, respectively, and $J_{0}$ and $J_{1}$ are Bessel functions. The constant $b$ is such that $b \rho_{0}$ is the smallest positive root of $J_{1}\left(b \rho_{0}\right)=0$. When $\lambda=0$, the two halves of the circular dipole are symmetric, and the vortex is asymmetric when $\lambda \neq 0$. In all cases, however, the dipole translates forward with constant velocity $U$.

Figure $4(a)$ shows the contours of the vorticity distribution inside the circular dipole for the symmetric case $(\lambda=0$, the Lamb dipole). The black lines in figure $4(a)$ show the five contours selected for a nested-contour model of the Lamb dipole. As noted previously, we assumed symmetry of the flow across the $x$-axis, so that the positively valued half of the core was modelled using five contours, and the contribution from the other half was derived from symmetry. Figure $4(b)$ shows the vorticity distribution along the centreline of the positively valued core of the Lamb dipole (dashed line), along with the piecewise-constant approximation obtained from the nested-contour model (solid line).

Figure 5 shows the evolution of the five-nested-contour model of the Lamb dipole, where the time and length have been normalised by $\rho_{0} / U$ and $\rho_{0}$, respectively. Initially, a small amount of irrotational fluid was entrained at the rear of the vortex (figure $5 b$ ). Eventually, the entrained fluid formed a thin cap near the forward stagnation point, which is visible in figure $5(d)$. A small amount of vortical fluid was also detrained into a small filament, which is seen trailing behind the dipole in figure $5(e)$. The formation of this tail of vorticity was also observed by van Geffen \& van Heijst (1998) in their viscous numerical simulation of the Lamb dipole, and deemed by the authors to have minimal influence on the motion of the vortex. Despite the formation 

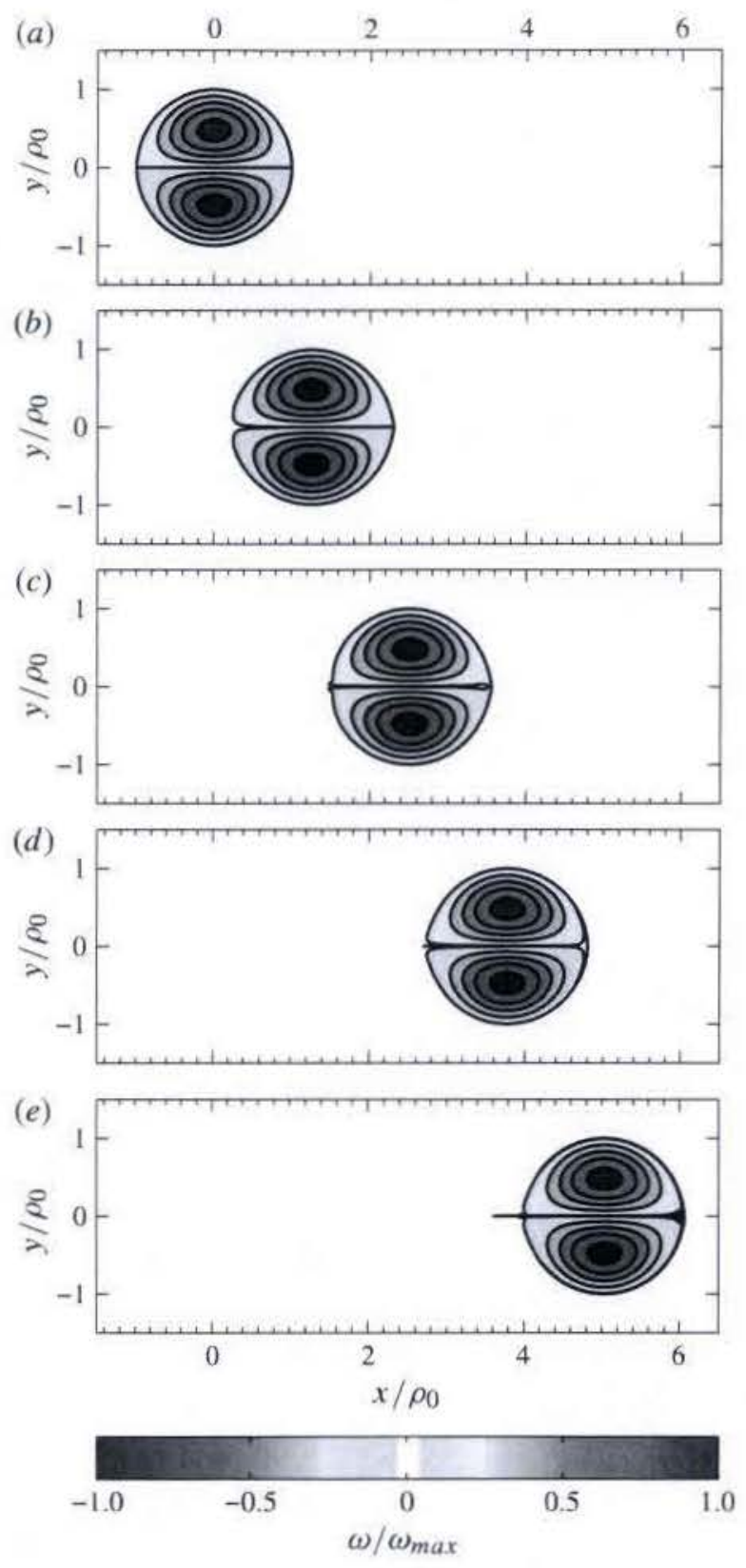

FIGURE 5. The evolution of a five-contour approximation to the Lamb dipole at $(a) t^{*}=$ $U t / \rho_{0}=0,(b) t^{*}=1.25,(c) t^{*}=2.5,(d) t^{*}=3.75$ and $(e) t^{*}=5$. The interior of the contours has been coloured by $\omega / \omega_{\max }$. The time and length have been normalised by the radius of the circular dipole $\left(\rho_{0}\right)$ and the translation velocity of the analytical solution $(U)$.

of these thin filaments, the model dipole retained a shape that closely resembled the analytical Lamb dipole. In addition, the vortex was found to have translated forward by an amount exceeding the expected $5 \rho_{0}$ by only $0.6 \%$ at $t^{*}=U t / \rho_{0}=5$ (figure $5 e$ ).

The accuracy of the nested-contour model for the Lamb dipole was found to improve when the number of contours used in the approximation was increased (figure 6). Figure 6(a) shows the circulation in one of the trailing filaments, expressed as a percentage of the circulation in the positive core of the analytical solution, as a function of the number of contours in the model. Similarly, figure $6(b)$ shows 

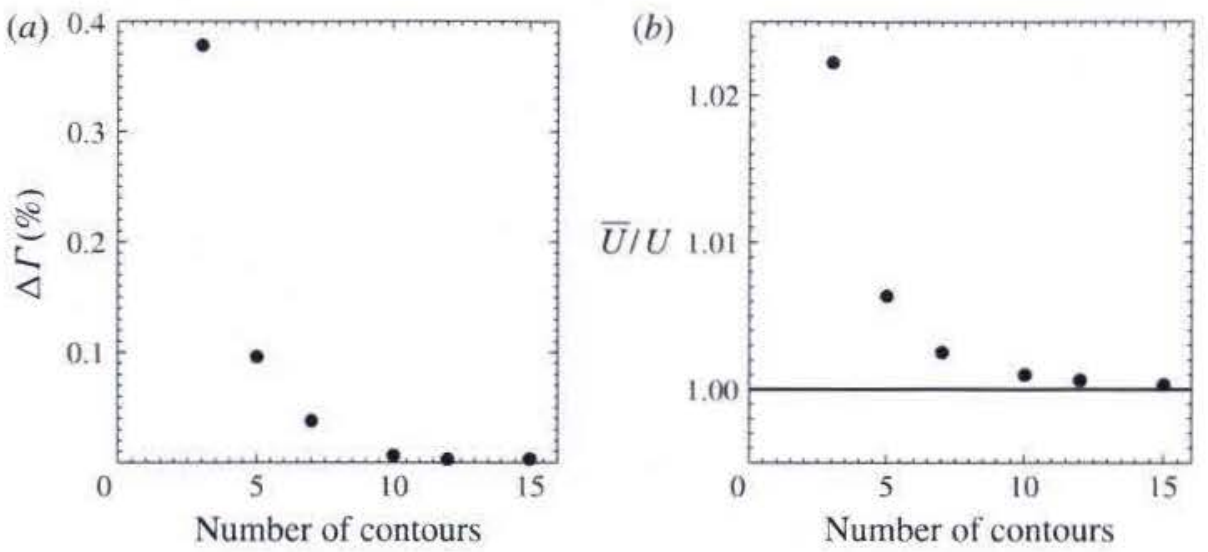

FIGURE 6. The effect of increasing the number of contours on the accuracy of the model. (a) Circulation in the vortex filament, expressed as a percentage of circulation in the analytical solution, as a function of the number of contours. (b) Comparison of the average translating velocity of the model dipole $(\bar{U})$ and the analytical value $(U)$, as a function of the number of contours.

a comparison of the average velocity of the model dipole $(\bar{U})$ and velocity of the Lamb solution $(U)$, for an increasing number of contours. When five contours were employed, the circulation in the filament was less than $0.1 \%$ of the core circulation, and the average velocity of the model dipole was within $1 \%$ of the Lamb value. It is clear from figure 6 that the nested-contour model constituted a good approximation to the Lamb dipole even for as few as five nested contours, and that the accuracy of the model increased with an increasing number of contours. In addition, figure 7 shows that the results for the five-contour model were largely insensitive to the specific numerical parameters used in the simulation. Figure $7(a)$ shows the normalised average velocity of the model dipole obtained from simulations employing different time steps, while figure $7(b)$ shows a plot of the average velocity for simulations where the maximum allowable length for the contour segments was varied.

\section{The construction of piecewise-linear models for axisymmetric vortex rings}

Multiple nested contours can also be used in axisymmetric flows to obtain piecewise-linear approximations to the vorticity distributions inside vortex rings. However, unlike in the two-dimensional case, no exact solutions to the Euler equations for a vortex ring are known, other than those considered by Hill (1894) and Norbury (1973). Therefore, we used several nested contours to construct models for axisymmetric vortex rings generated experimentally using a piston-cylinder arrangement.

For this purpose, we considered the vortex rings generated in the long-stroke-ratio $(L / D=12)$ starting jet analysed in O'Farrell \& Dabiri $(2010)$. Our objective was to obtain nested-contour models for the vortex ring at different stages of its growth, until its saturation and pinch-off. Therefore, we constructed models for vortex rings with stroke ratios $(L / D)_{\text {desired }}=1,2,3$ and 4 . Rather than conducting four different experiments to generate isolated vortex rings at these stroke ratios, we extracted from different time instants of the $L / D=12$ data the vortex rings for which the total circulation matched the circulation expected in isolated vortex rings of the desired stroke ratio. 

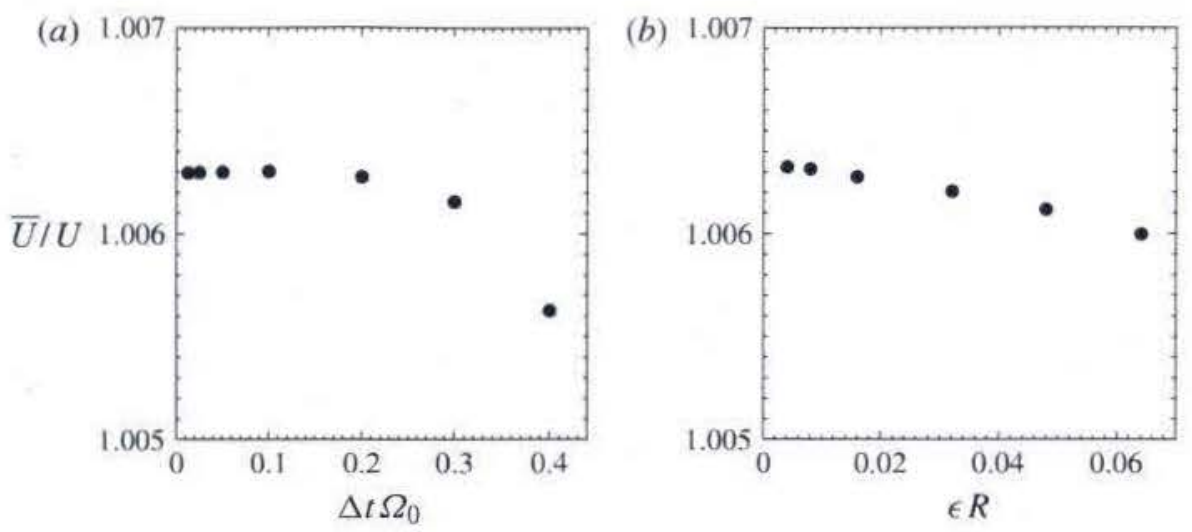

FIGURE 7. The sensitivity of the simulation to the numerical parameters. Comparison of the average translating velocity of the model dipole $(\bar{U})$ and the analytical value $(U)$ as a function of $(a)$ the size of the time step and $(b)$ the maximum segment length $\epsilon$.

When looking at the time history of the $L / D=12$ data, we considered that if fluid ejection were halted at a non-dimensional time of $\hat{T}=\bar{U}_{p} t / D=(L / D)_{\text {desired }}$, the resulting isolated vortex ring would have a circulation equal to the total circulation ejected by the apparatus up to $\hat{T}=(L / D)_{\text {desired }}$, which we called $\Gamma_{\text {desired }}$. However, all of $\Gamma_{\text {desired }}$ need not be found in the leading vortex immediately after $\hat{T}=(L / D)_{\text {desired }}$; some of it may remain in the trailing jet and be absorbed by the ring some time later. Figure 8 shows the time histories of the total circulation emanating from the apparatus $(\Gamma)$, as well as the circulation in the leading vortex ring $\left(\Gamma_{\text {ring }}\right)$. In the figure, $\Gamma_{\text {ring }}$ is identical to the total circulation until $\hat{T} \approx 2$. For $\hat{T}>2$, the vortex ring advected away from the nozzle, while remaining attached to the shear layer and continuing to accept circulation from it (Gharib et al. 1998; Gao \& Yu 2010; Domenichini 2011). As a result of this separation of the vortex ring from the nozzle edge, vortical fluid ejected after $\hat{T} \approx 2$ was not immediately absorbed by the vortex, and $\Gamma_{\text {ring }}$ increased at a rate slower than the total circulation.

Consequently, for $(L / D)_{\text {desired }}>2$, the leading vortex ring at $\hat{T}=(L / D)_{\text {desired }}$ did not yet contain the same circulation as an isolated vortex ring with the desired stroke ratio. Therefore, it was not sufficient to look at instantaneous contours of vorticity at $\hat{T}=(L / D)_{\text {desired }}$ to obtain model vortex rings at these desired stroke ratios. Rather, we needed to determine the time instant at which the leading vortex ring in the $L / D=12$ data achieved the desired circulation $\Gamma_{\text {desired }}$. We achieved this by comparing $\Gamma_{\text {ring }}(\hat{T})$ for the $L / D=12$ data with the time history of the total circulation $(\Gamma(\hat{T}))$, to find the $\hat{T}_{\text {model }}$ at which the circulation in the leading vortex ring equalled the total circulation at $\hat{T}=(L / D)_{\text {desired }}$ (i.e. we searched for $\hat{T}_{\text {model }}$ such that $\left.\Gamma_{\text {ring }}\left(\hat{T}_{\text {model }}\right)=\Gamma_{\text {desired }}\right)$. We then used instantaneous contours of vorticity at $\hat{T}_{\text {model }}$ to model the vortex ring with $(L / D)_{\text {desired }}$.

The annotations and arrows in figure 8 illustrate this process for $(L / D)_{\text {desired }}=4$, where $\hat{T}_{\text {model }}$ was found to be 7.8 . We first found the total circulation at $\hat{T}=$ $(L / D)_{\text {desired }}=4\left(\Gamma_{\text {desired }}=38 \mathrm{~cm}^{2} \mathrm{~s}^{-1}\right)$ and then searched for the $\hat{T}_{\text {model }}$ at which the circulation in the leading vortex ring was equal to $\Gamma_{\text {desired }}$.

Having determined $\hat{T}_{\text {model }}$, we constructed a nested-contour model by extracting contours of the vorticity density $(\xi=\omega / r)$ at $\hat{T}=\hat{T}_{\text {model }}$ for the leading vortex in 


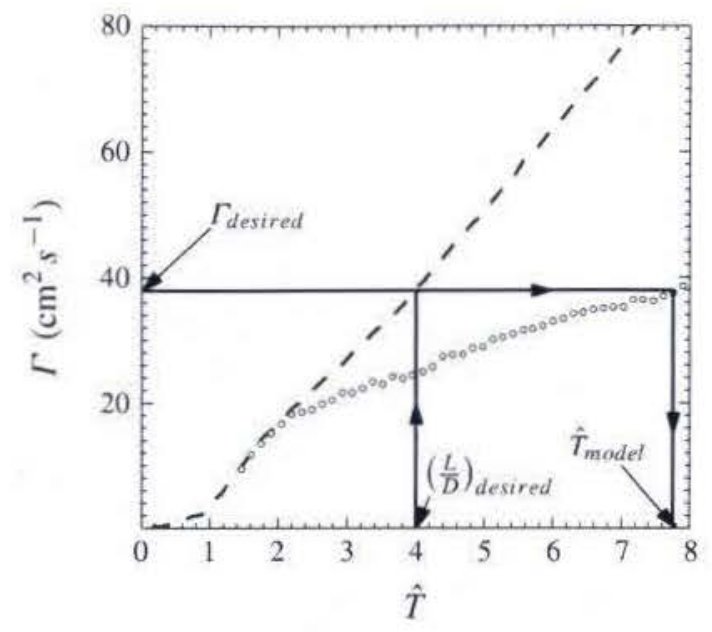

FigurE 8. The total circulation emanating from the nozzle (--) and the vortex ring circulation (o) as a function of the formation time $\hat{T}$ for an experimentally generated vortex ring. The arrows illustrate the method used to determine $\hat{T}_{\text {model }}$ when modelling a vortex ring with a stroke ratio of 4 . The total circulation emanating from the nozzle at $\hat{T}=4$ was compared with the time history of the vortex ring, to determine the $\hat{T}$ at which all of the circulation ejected by $\hat{T}=(L / D)_{\text {desired }}=4\left(\Gamma_{\text {desired }}\right)$ had been absorbed by the vortex ring.

the experimental data. Figure $9(a)$ shows contours of vorticity at $\hat{T}=\hat{T}_{\text {model }}=7.8$. In order to apply the axisymmetric contour dynamics algorithm described in $\$ 2.1$, our models were required to consist of nested regions with linear distributions of vorticity $\omega_{m}=\sum_{j=1}^{m} \Omega_{j} r$ (where $m \in[1, \ldots, n]$ ). Therefore, we constructed our models by extracting contours of constant $\xi$ from the experimental data, and assuming the value of $\xi$ within each contour to have a constant value $\xi_{m}=\sum_{j=1}^{m} \Omega_{j}$ (where $m \in[1, \ldots, n]$ ). Figure $9(b)$ shows five instantaneous contours of vorticity density at $\hat{T}=7.8$, used to construct a five-nested-contour model for a vortex ring with a stroke ratio of 4 . The small regions of high $\xi$ near the symmetry axis between $x / D \approx 4$ and $x / D \approx 6$ (which are absent in the contours of $\omega$ in figure $9 a$ ) are due to noise in the vorticity field, which is amplified when divided by small values of $r$.

Figure 10 shows these same contours of $\xi$, along with the five-contour model of the vortex which was constructed from these contours (in red). The vorticity density $\xi$ in the leading vortex ring core at $\hat{T}=7.8$ (shown in figure 10) was averaged with the distributions of $\xi$ in the cores at the two adjacent time steps, in order to provide a smoother distribution. The vorticity densities around the cross-sections of the ring in the positive $r$ half-plane and the negative $r$ half-plane were also averaged to increase the smoothness of the contours in the model. Five contours of constant $\xi$ were then extracted from the averaged vorticity density, and used to construct the model shown in red in figure 10. Because the vortex ring remained attached to the shear layer, the rear boundary of the ring was not easily distinguishable. However, isolated laminar vortex rings are known from flow visualisation experiments to exhibit high degrees of fore-aft symmetry (Lim \& Nickels 1995). Therefore, we reflected the contour shapes obtained for the front half of the vortex about the core centreline, and constructed the symmetric core shown in figure 10.

A comparison of the distributions of $\xi$ and $\omega$ along the centreline of the vortex core in the experimental and model vortex rings is shown in figure 11. Since in the model 

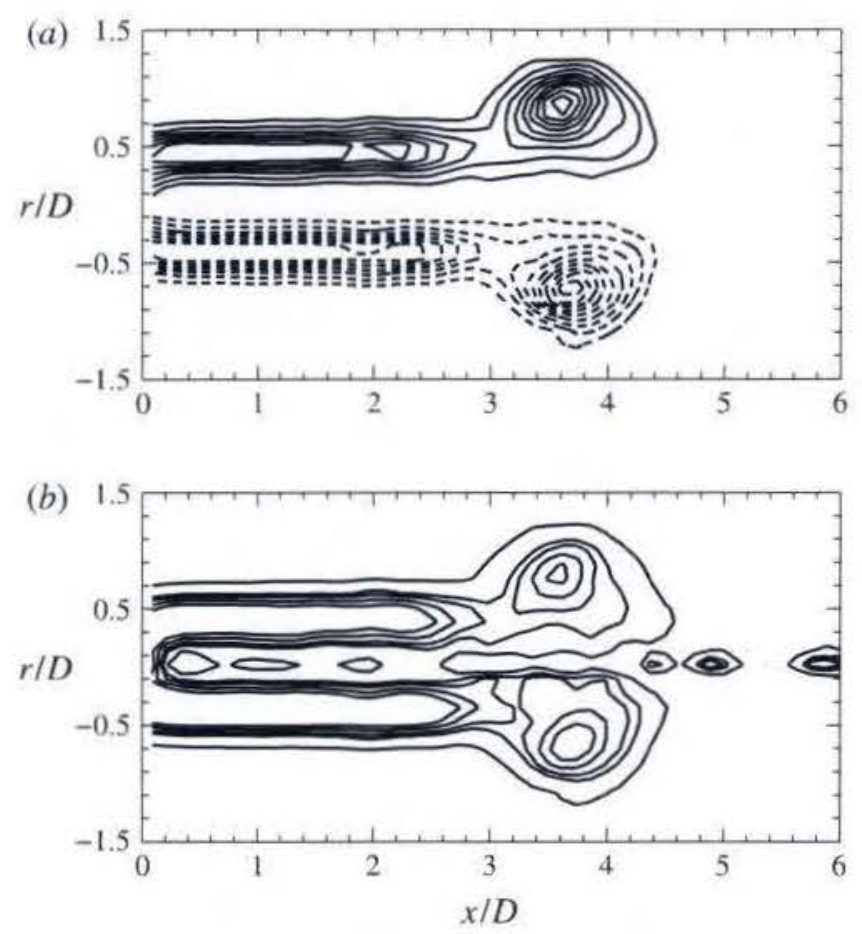

FIGURE 9. Contours of vorticity and vorticity density at $\hat{T}=7.8$, the time instant at which all of the vorticity generated by $\hat{T}=4$ had been accepted by the vortex ring. (a) Contours of $\omega$. Levels: $\min =10 \%$ of $\omega_{\max }$, $\max =90 \%$ of $\omega_{\max }$, increment $=10 \%$. Negative values are denoted by the dashed lines. (b) Contours of $\xi / \xi_{\max }$, where $\xi_{\max }$ is the maximum $\xi$ inside the cores. Levels: $0.09,0.36,0.49,0.68,0.88$. Flow is from left to right.

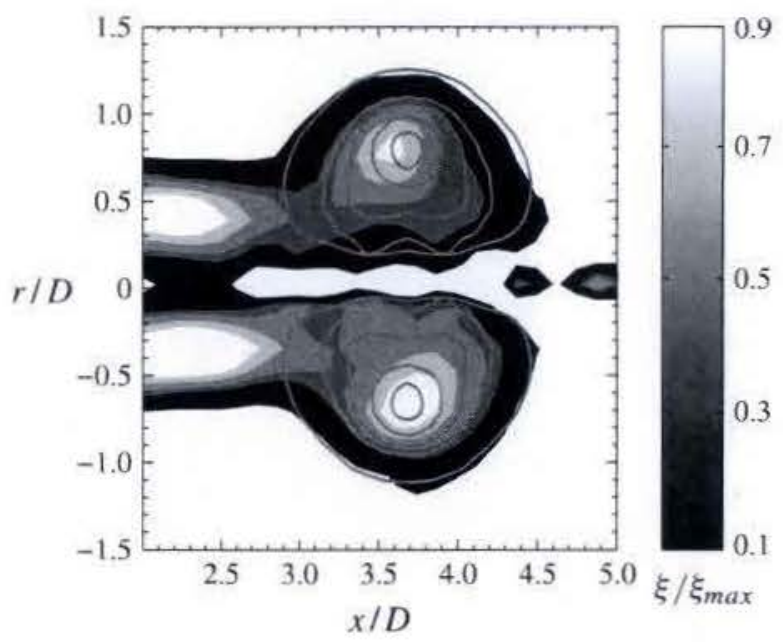

FIGURE 10. Contours of $\xi / \xi_{\max }$ at $\hat{T}=7.8\left((L / D)_{\text {desired }}=4\right)$ and the superimposed fivecontour model of a vortex ring with a stroke ratio of 4 (in red). Contour levels: 0.09 , $0.36,0.49,0.68,0.88$. The direction of flow and the direction of propagation of the model vortex ring coincide with the direction of increasing $x$.

vortex ring the vorticity density was assumed to be constant inside each contour $\left(\xi_{m}=\right.$ $\sum_{j=1}^{m} \Omega_{j}$, where $\left.m \in[1, \ldots, n]\right)$, the experimental distribution of $\xi$ was approximated 

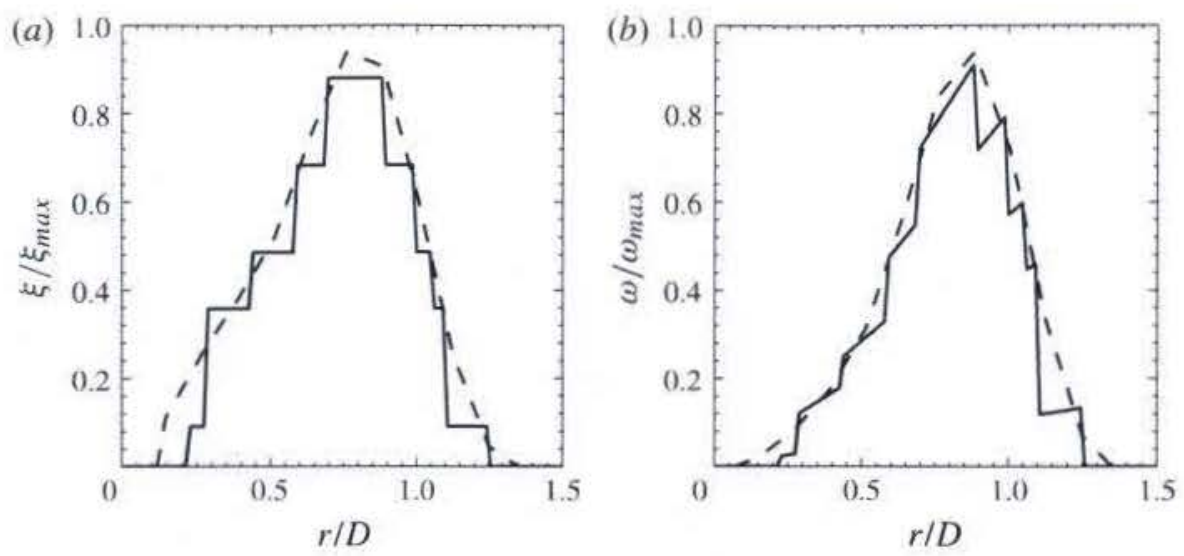

FIGURE 11. (a) Experimental (--) and five-contour model (-) distribution of $\xi / \xi_{\max }$ along the centreline of one of the cores of the vortex ring. (b) Experimental (--) and five-contour model $(-)$ vorticity distribution along the centreline of one of the cores of the vortex ring.

by a series of steps (figure $11 a$ ) in the model. However, the vorticity inside each contour in the model vortex was given by $\omega_{m}=\xi_{m} r=\sum_{j=1}^{m} \Omega_{j} r$ (where $m \in[1, \ldots, n]$ ). Hence, the model vorticity tracked the experimental vorticity well on the half of the core closest to the symmetry axis, where $\omega$ had a positive slope in both cases $(r / D<$ 0.85 in figure $11 b$ ). However, where the experimental vorticity was decreasing in $r$, the model $\omega$ (which was constrained to have a positive slope) had a jagged shape $(r / D>0.85$ in figure $1 / b)$.

\section{The search for steady-state models for experimental vortex rings}

Using the numerical scheme described in $\$ 2.1$, we simulated the evolution of the nested-contour model for the vortex ring with a stroke ratio of 4 shown in figure 10 . Figure 12 shows the evolution of the vortex ring as a function of the normalised time $t^{*}=\bar{U}_{p} t / D$, where $\bar{U}_{p}$ and $D$ are the time-averaged piston velocity and nozzle diameter from the experimental data, respectively. Since the model was seen to detrain the circulation into a tail of considerable size, the five contours obtained directly from the experimental data did not comprise a steady solution of the Euler equations. This is not unexpected, for a number of reasons. At least a small amount of detrainment is expected, given our findings in modelling the Lamb dipole. In the case of the Lamb dipole, however, the vorticity distribution was an exact solution to the Euler equations. In the axisymmetric case, the vorticity distribution being modelled was obtained from a viscous case $(\operatorname{Re}=1400)$, while the contour dynamics algorithm solves the inviscid vorticity equation. Therefore, the contours obtained from the experiment were not expected to closely approximate an inviscid solution.

At the stage shown in figure 12(e), the computational costs associated with resolving the stretching of the tail made continuation of the simulation prohibitively expensive. Continuation of this calculation in its current state would have required implementation of the contour surgery algorithm developed by Dritschel $(1988 a)$, which implied additional computational cost and a large increase in the complexity of the algorithm. However, Pozrikidis (1986) was able to continue the simulation of the evolution of perturbed spherical vortices after filament formation by excision of the vortex filaments. Furthermore, Pozrikidis (1986) found this simplification to 

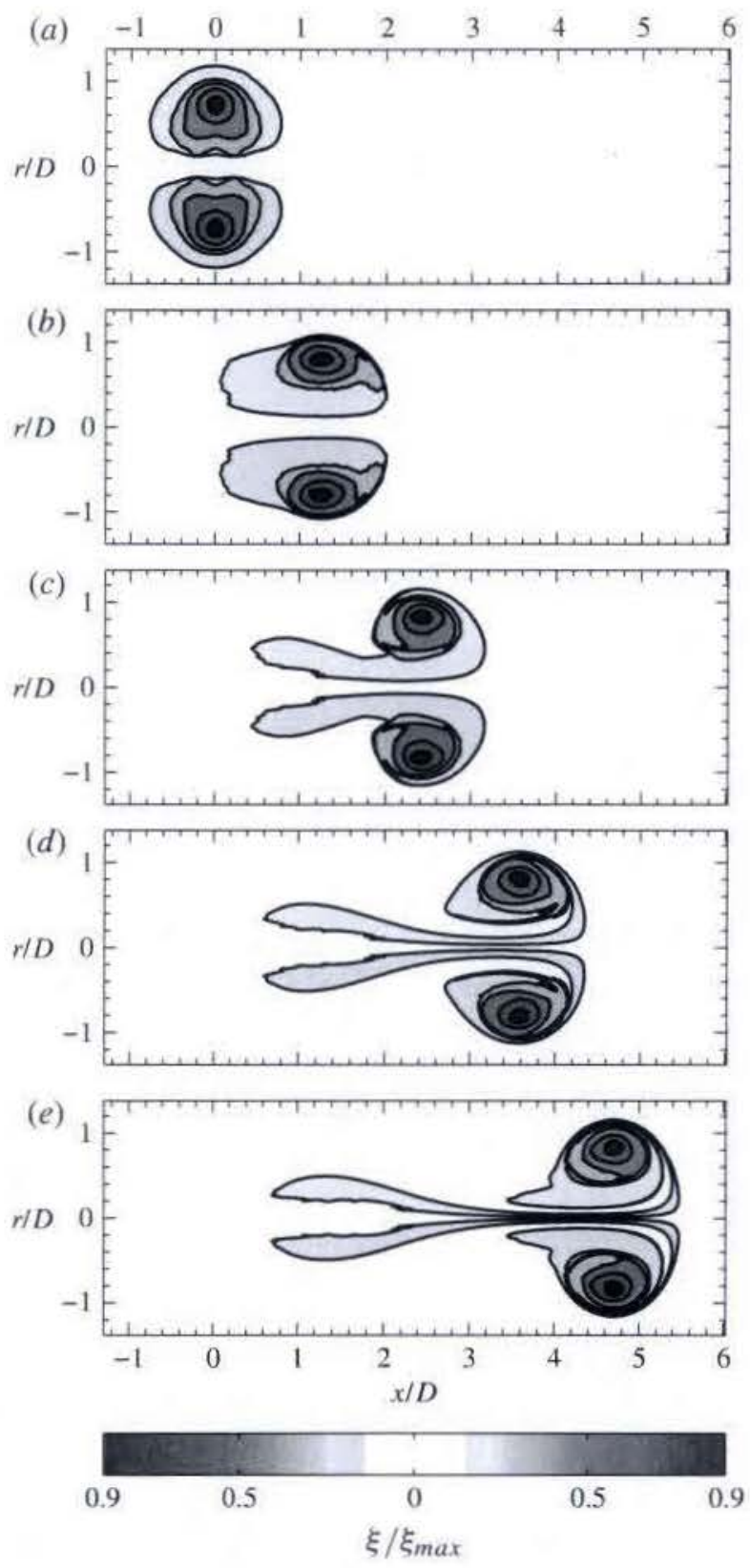

FIgURE 12. The evolution of a five-contour model of a vortex ring with $L / D=4$, at (a) $t^{*}=\bar{U}_{p} t / D=0,(b) t^{*}=2.1,(c) t^{*}=4.3,(d) t^{*}=6.5$ and $(e) t^{*}=8.6$. Inside each contour, $\xi=\omega / r=\Omega_{j}$ is constant, and the interior of the contours has been coloured by $\xi / \xi_{\max }$, where $\xi_{\max }$ is the maximum vorticity density in the experimental data.

have a negligible effect on the accuracy of the simulations of the evolution of the remaining vorticity, and the same method was successfully applied in O'Farrell \& Dabiri (2012). Since the vortex tail in figure 12(e) was not of particular interest, we adopted the strategy described in Pozrikidis (1986) and excised the tail at $t^{*}=8.6$. We then continued the simulation of the vortex without the tail.

Panels $(a)-(e)$ of figure 13 show the evolution of the model vortex ring after the excision of the tail. Once again, the vortex was found to shed circulation into a tail 

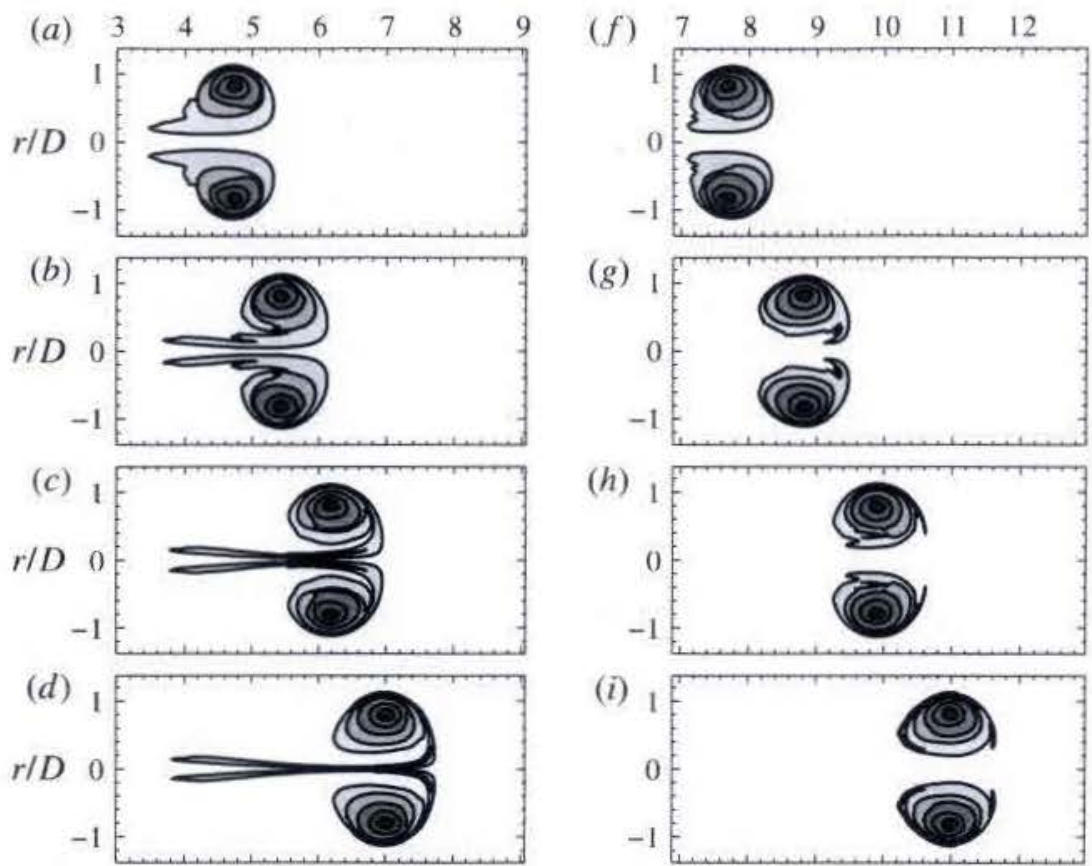

(i)
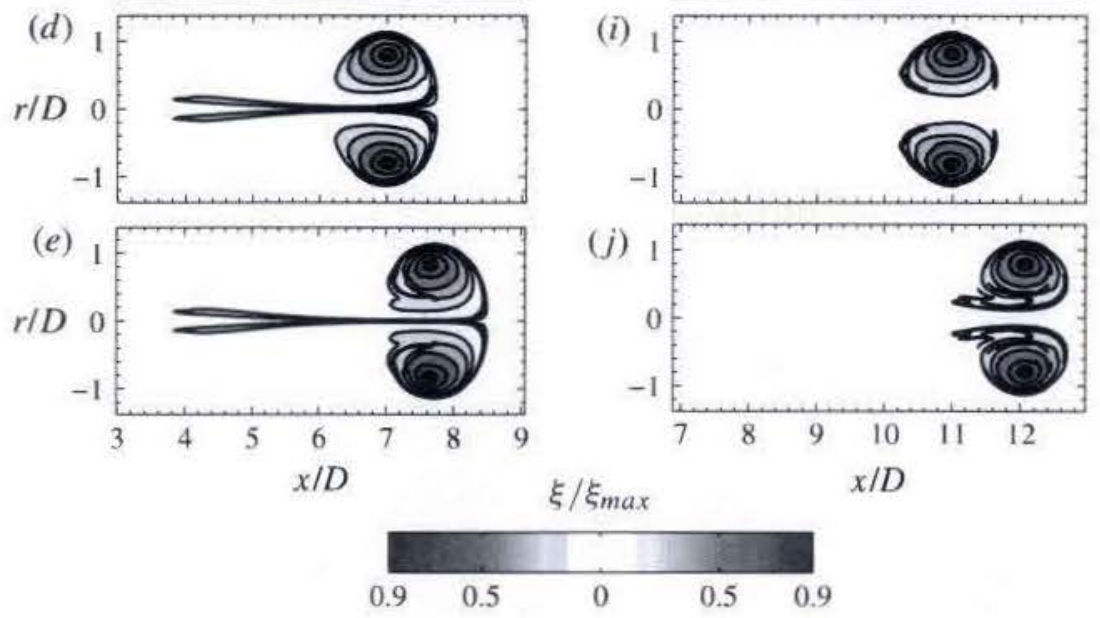

FIGURE 13. The evolution of a five-contour model of a vortex ring with $L / D=4$ after filament excision, at (a) $t^{*}=8.7,(b) t^{*}=10.1,(c) t^{*}=11.6,(d) t^{*}=13.1$ and $(e) t^{*}=$ 14.5, and after the second filament excision, at $(f) t^{*}=14.6,(g) t^{*}=16.7,(h) t^{*}=18.8$, (i) $t^{*}=21$ and $(j) t^{*}=23.1$. Inside each contour, $\xi=\omega / r=\Omega_{j}$ is constant, and the interior of the contours has been coloured by $\xi / \xi_{\max }$, where $\xi_{\max }$ is the maximum vorticity density in the experimental data.

that trailed behind the ring. However, the amount of circulation shed by the vortex ring in this case was significantly reduced. A second excision of the vorticity in the tail allowed the simulation to continue to longer times. The evolution of the vortex ring after the second excision is shown in panels $(f)-(j)$ of figure 13 , where the formation of filaments which wrapped around the vortex core was observed, but no vorticity was detrained into a tail. The formation of filaments has been observed in previous contour dynamics studies under a variety of conditions (Deem \& Zabusky 1978; Pozrikidis 1986; Ye \& Chu 1995; O'Farrell \& Dabiri 2012), and it is known to occur wherever there are irregularities or regions of local concavity in the contour outline (O'Farrell \& Dabiri 2012).

We repeated this excision process several times, and monitored the evolution of the vortex after each excision. Figure 14 shows the decrease in the vortex ring circulation after each excision, expressed as a percentage of the circulation immediately preceding the excision. It is clear from this figure that the amount 


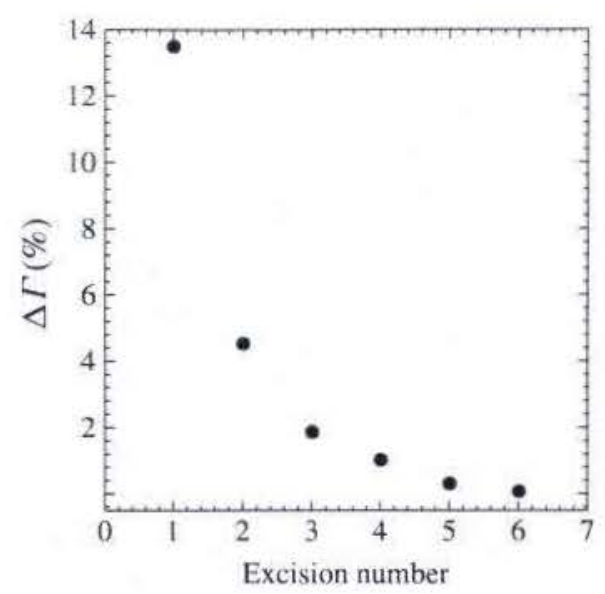

FIGURE 14. The circulation in the vortex filament immediately before each excision, expressed as a percentage of the vortex circulation before the excision.

of circulation shed by the vortex decreased after each excision. Furthermore, the vortex appeared to be approaching an asymptotic steady state, since the decrease in circulation was under $0.1 \%$ by the sixth excision and the model vortex was found to approach a steady translation velocity. Therefore, we used the configuration of the five-contour-approximation after the sixth excision as our model for vortex rings with a stroke ratio of 4 .

The method outlined in $\S 4$ was used to extract nested-contour approximations for vortex rings with stroke ratios of $L / D=1,2,3,4$ from the experimental data. Each of these models was evolved numerically in time as outlined in the preceding paragraphs, with filaments being excised as needed, until their asymptotic steady shape was determined. Figure 15 shows the resulting five-nested-contour models for vortex rings at stroke ratios of $1,2,3$ and 4 . These models comprised a family which was used to model vortex rings at different stages of their growth. Following Pozrikidis (1986), Ye \& Chu (1995) and our work on the Norbury and Pierrehumbert families of vortices (O'Farrell \& Dabiri 2012), we therefore investigated the perturbation response of this family of vortex rings.

\section{The perturbation responses of model vortex rings}

In order to study the perturbation responses of model vortex rings in a starting jet at different stages in their growth, and their relationship to pinch-off, we considered a type of perturbation that simulated the addition of vorticity from a trailing shear layer. The perturbation involved the addition of a small amount of circulation at the rear of the vortex by perturbing the shape of the outermost contour. The perturbation to the outer contour had the following form:

$$
x^{\prime}= \begin{cases}x\left[1+d \sin \left(\frac{r-r(\pi)}{w} \pi\right)\right], & \pi \leqslant \eta \leqslant \eta_{w} . \\ x, & \text { elsewhere, }\end{cases}
$$

where $x^{\prime}$ was the perturbed coordinate, $x$ and $r$ were the unperturbed coordinates and $\eta$ was the polar angle as defined in figure 16. The $r$ coordinate was unchanged, so the perturbation amounted to inserting a tail of vorticity of length $d$ and width $w$ at the 


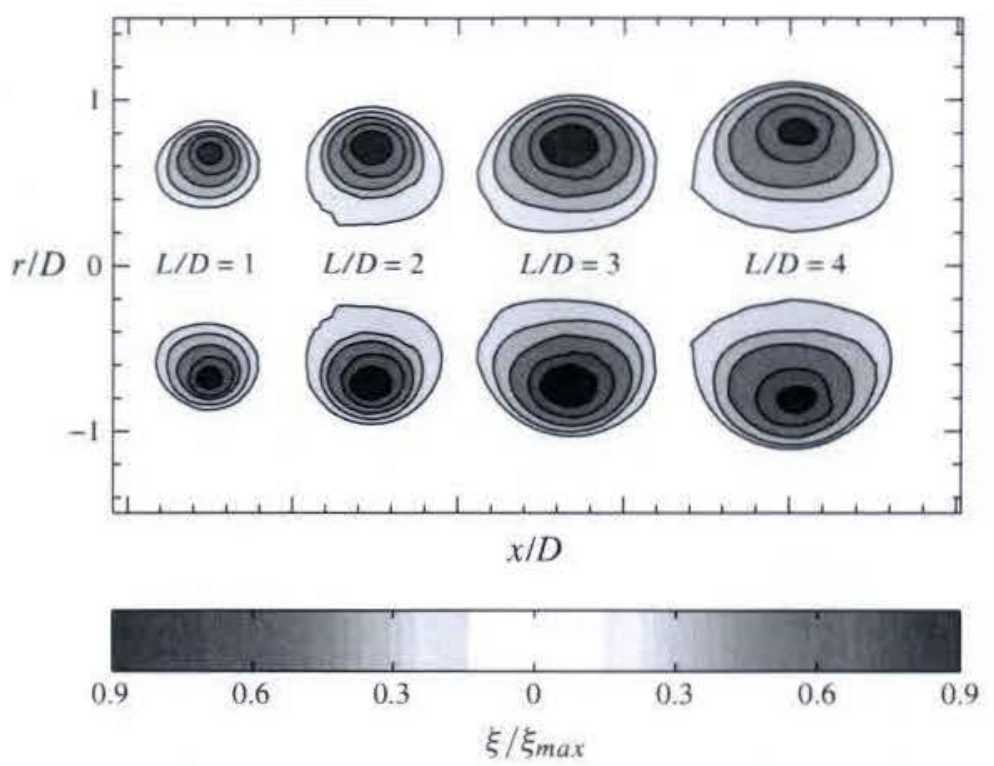

FIgURE 15. Models for vortex rings with $L / D=1,2,3,4$, constructed using five nested regions of constant $\xi=\omega / r=\Omega_{j}$. The regions are coloured by $\xi / \xi_{\max }$, where $\xi_{\max }$ is the maximum vorticity density inside the cores in the experimental data.

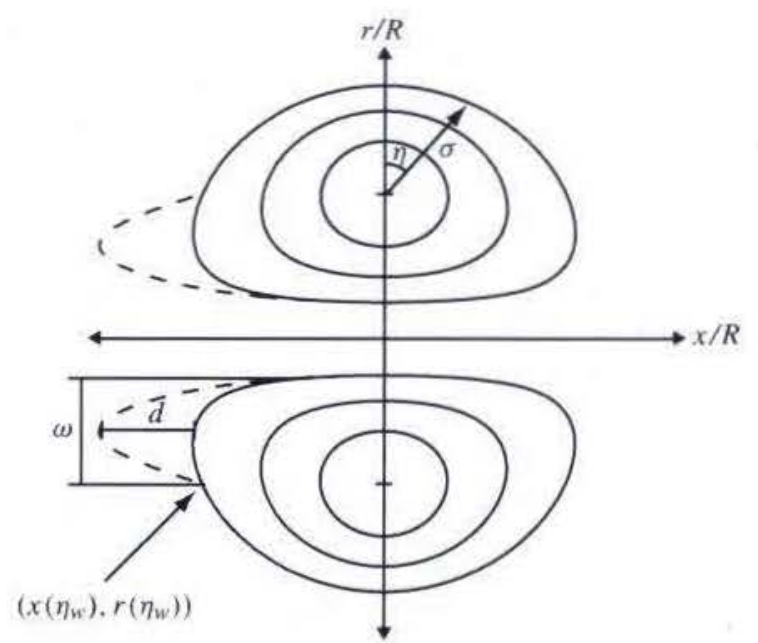

FIGURE 16. Perturbation simulating a trailing shear layer, for a three-contour vortex. The unperturbed vortex is depicted by the solid lines. The dashed lines represent a perturbation of the type defined in $(6,1)$ to the outer contour of a model vortex. The vortices propagate from left to right.

rear of the vortex. Here, $\eta_{w}$ was the polar angle at which $r=r(\pi)+w$, as indicated in figure 16. We considered perturbations where $w=2 R / 3$, and $d$ was varied in order to investigate the change in the perturbation response of the vortices as the amount of circulation introduced was increased.

The perturbation scheme introduced here was designed to mimic the pinch-off of experimentally generated vortex rings. However, we have found that quantitative changes in the details of the perturbation do not change the qualitative results for the perturbation response. Similar behaviour was previously observed in the perturbation response of the Norbury family, where we found that the details of the 

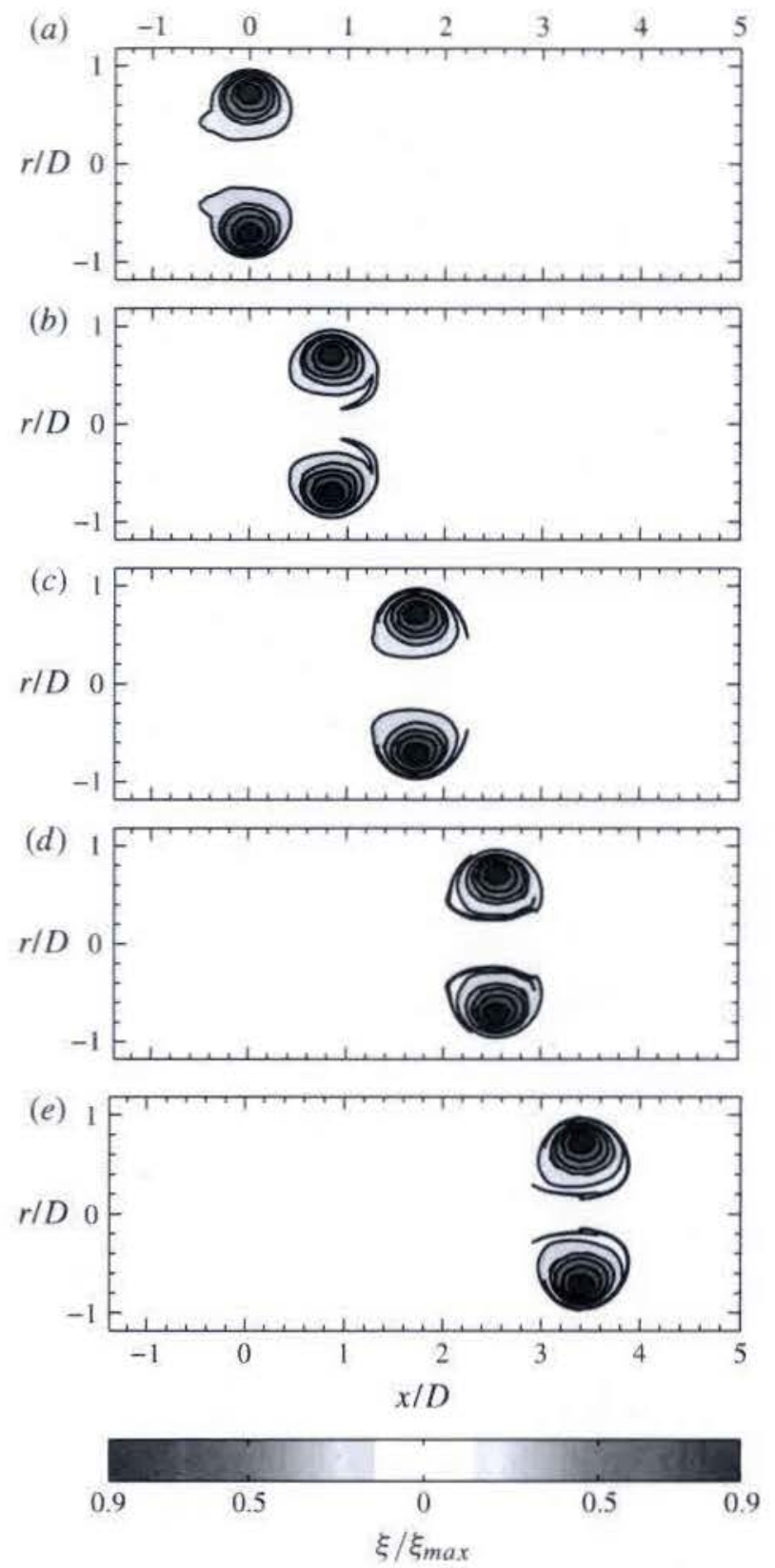

FIGURE 17. The evolution of a five-contour model of a vortex ring with $L / D=2$, subjected to a perturbation containing $2 \%$ of the unperturbed circulation, at $(a) t^{*}=0$, (b) $t^{*}=2.7,(c) t^{*}=5.4,(d) t^{*}=8.1$ and $(e) t^{*}=10.8$.

perturbations introduced to the family had little effect on the qualitative response (O'Farrell \& Dabiri 2012).

We introduced perturbations of the form described above to the four model vortices in figure 15, and simulated the evolution of the perturbed vortices using the method described in $\$ 2.1$. The size of the perturbations was quantified by the amount of circulation added to the vortex ring by the perturbation, expressed as a percentage of the circulation in the unperturbed vortex ring $(\Delta \Gamma / \Gamma(\%))$. Figure 17 depicts the evolution of a model vortex ring with a stroke ratio of 2 , subjected to a perturbation containing $2 \%$ of the unperturbed vortex circulation. The tail introduced by the 

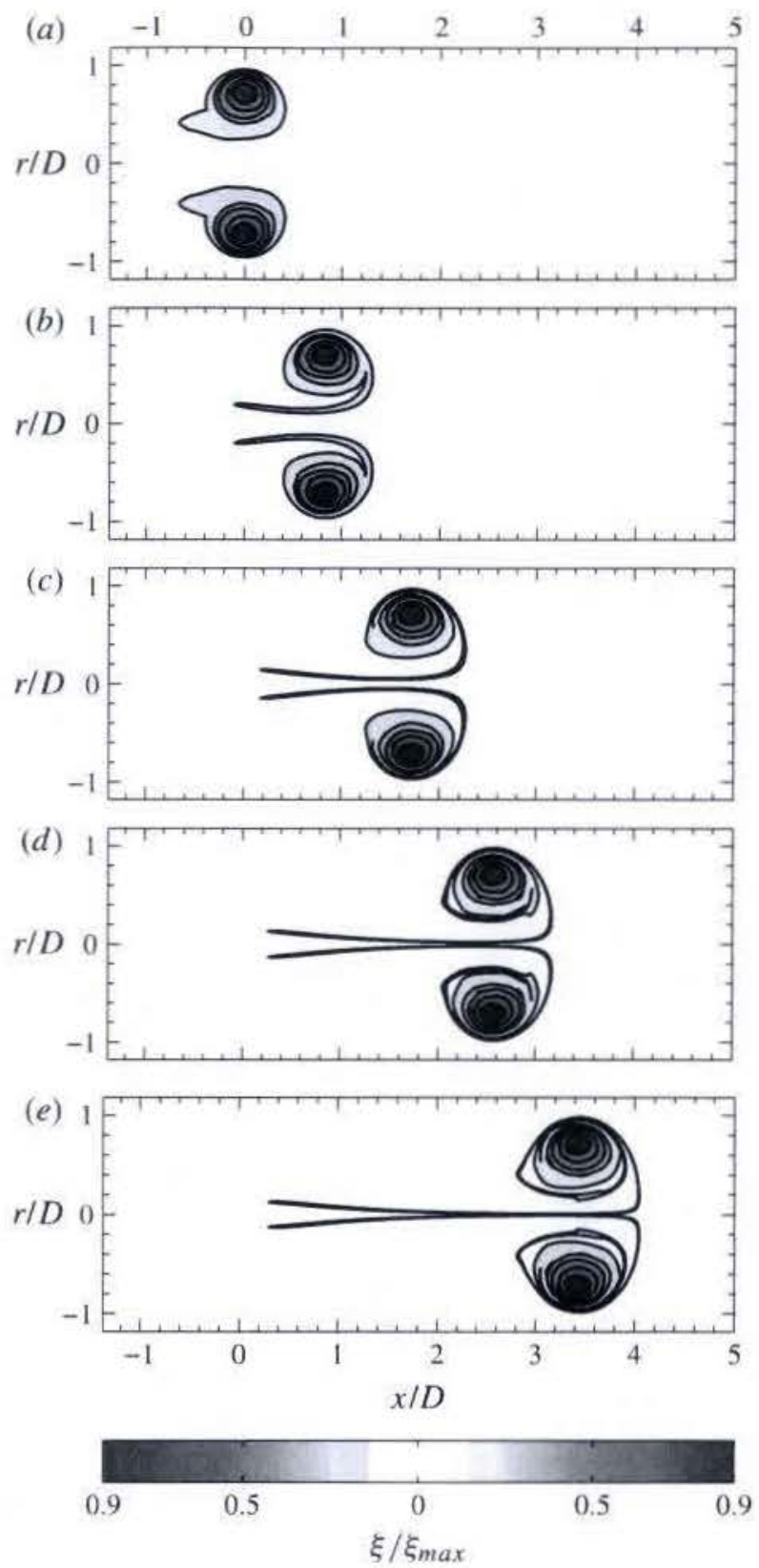

FIGURE 18. The evolution of a five-contour model of a vortex ring with $L / D=2$, subjected to a perturbation containing $4 \%$ of the unperturbed circulation, at (a) $t^{*}=0$, (b) $t^{*}=2.7,(c) t^{*}=5.4,(d) t^{*}=8.1$ and $(e) t^{*}=10.8$.

perturbation was seen to develop into a vortex filament, much like those observed previously when perturbing sufficiently thin-cored members of the Norbury family (Ye \& Chu 1995; O'Farrell \& Dabiri 2012). Like in those cases, the filament was found to wrap around the vortex core and continue to move with the vortex ring, with no detrainment of vorticity into a tail.

When the size of the perturbation was increased, however, we noticed a change in the response of the model vortex ring with $L / D=2$. Figure 18 shows the evolution of the model vortex ring with a stroke ratio of 2 , when subjected to a perturbation containing $4 \%$ of the unperturbed vortex circulation. In this case, the vortex ring was 

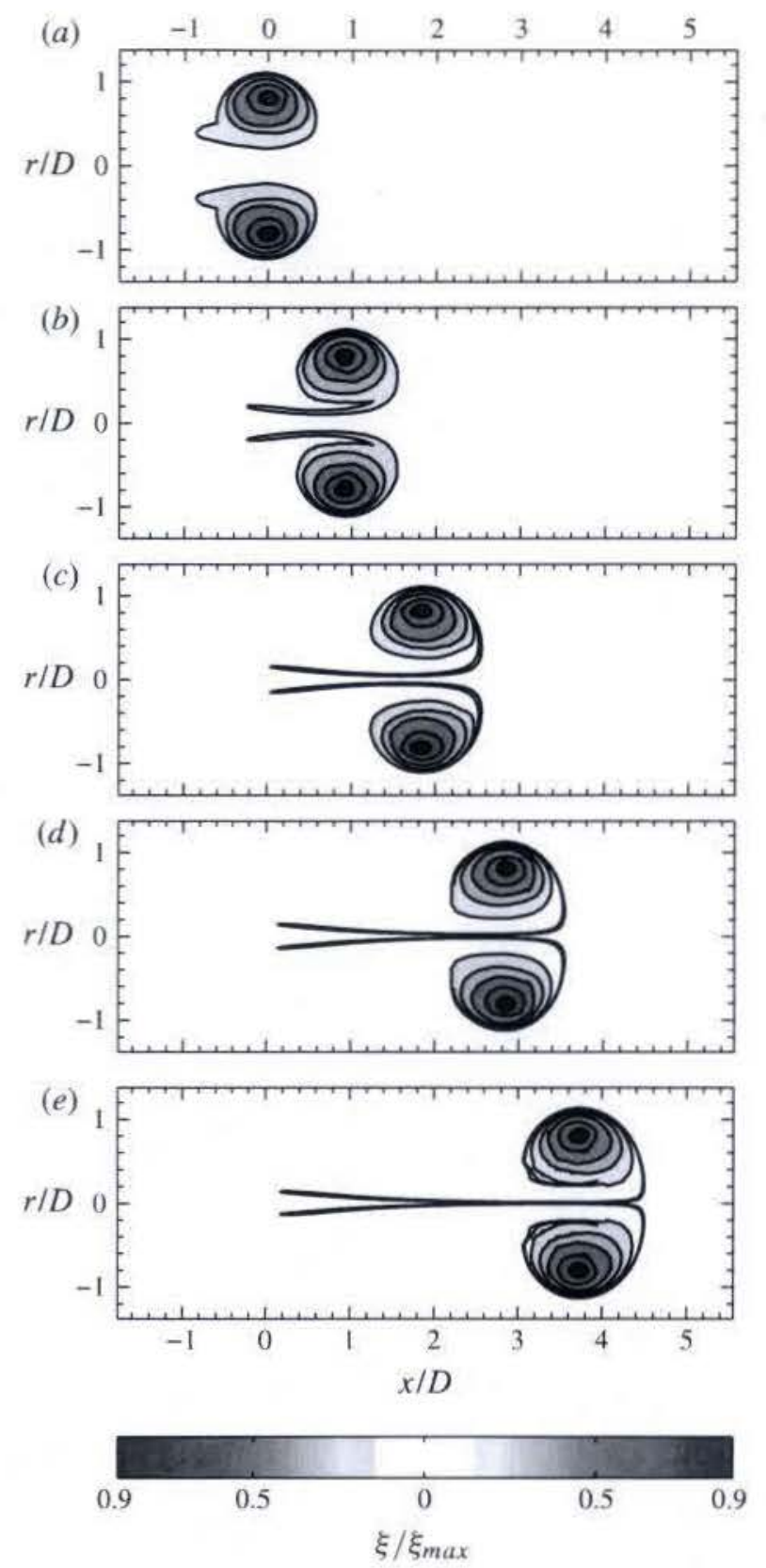

FIGURE 19. The evolution of a five-contour model of a vortex ring with $L / D=4$, subjected to a perturbation containing $2 \%$ of the unperturbed circulation, at $(a) t^{*}=0$, (b) $t^{*}=1.9$, (c) $t^{*}=3.8$, (d) $t^{*}=5.7$ and $(e) t^{*}=7.6$.

found to detrain vorticity from the outermost contour into a tail that lingered behind the ring.

For the model vortex ring with a stroke ratio of 4 , the change in perturbation response was found to occur at a perturbation size smaller than any of those introduced to the vortex with $L / D=2$. The evolution of a vortex ring with $L / D=4$ subjected to a perturbation containing $2 \%$ of the unperturbed vortex circulation is shown in figure 19. The shedding of vorticity into a tail was apparent in this vortex even for perturbations containing only $2 \%$ of the unperturbed vortex circulation, 


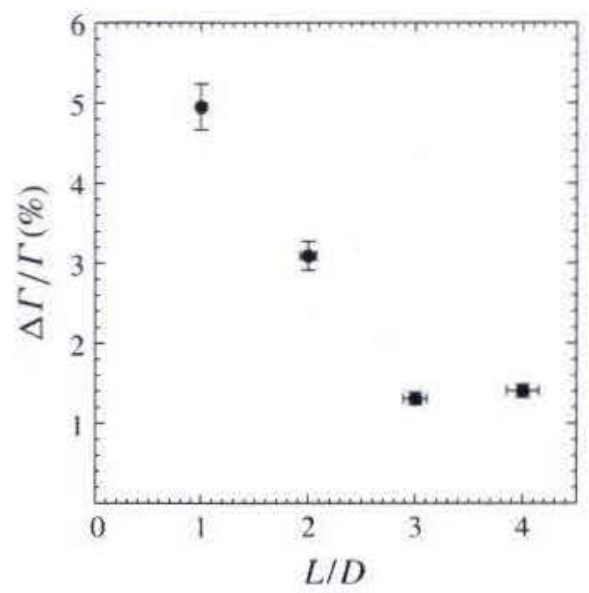

FIGURE 20. The maximum perturbation size accepted by the model vortex, as a function of the vortex stroke ratio. The perturbation size was quantified by the circulation added to the vortex $(\Delta \Gamma)$, expressed as a percentage of the vortex circulation.

which had not been observed to lead to tail shedding in the vortex with $L / D=2$ (figure 17).

The fact that the vortex ring with $L / D=2$ was able to withstand larger perturbations than the vortex with $L / D=4$ without detraining vorticity into a tail suggests that the response of the model vortices to this type of perturbation is consistent with the occurrence of pinch-off in starting jets. We therefore determined the maximum perturbation size that the four model vortex rings could accept before detraining vorticity into a tail. These results are shown in figure 20 , and indicate that the amount of circulation the model vortex rings could accept before shedding a tail decreased with increasing stroke ratio, before levelling off at a stroke ratio of approximately 3 .

For small stroke ratios, a decrease in the maximum allowable perturbation size before shedding was expected, as the model vortex rings grew closer to a limiting size known to exist from the experiments of Gharib et al. (1998) and others (Dabiri \& Gharib 2004; Krueger, Dabiri \& Gharib 2006; Pawlak et al. 2007). At a stroke ratio of approximately 3 , this decrease in the maximum perturbation size stopped, indicating that the stroke-ratio- 3 and -4 vortex rings were comparably close to the limiting size.

The threshold perturbation size for each model was found to be largely insensitive to the specific numerical parameters used in the simulation. For example, figure 21 shows that the maximum perturbation size accepted by the $L / D=4$ model remained within $1 \%$ of the asymptotic value when $\Delta t<2.5 / \Omega_{0}$. The results were even more insensitive to changes in the maximum allowable segment length.

\section{Conclusions}

We constructed models for vortex rings and vortex dipoles using several nested patches of vorticity, and simulated their evolution using contour dynamics methods. The use of nested contours allowed us to construct more realistic approximations to vortex rings and dipoles, while retaining the computational simplicity of the inviscid contour dynamics method.

In two dimensions, we constructed nested-contour models for the Lamb dipole, and found their agreement with the analytical solution to be excellent, even for 


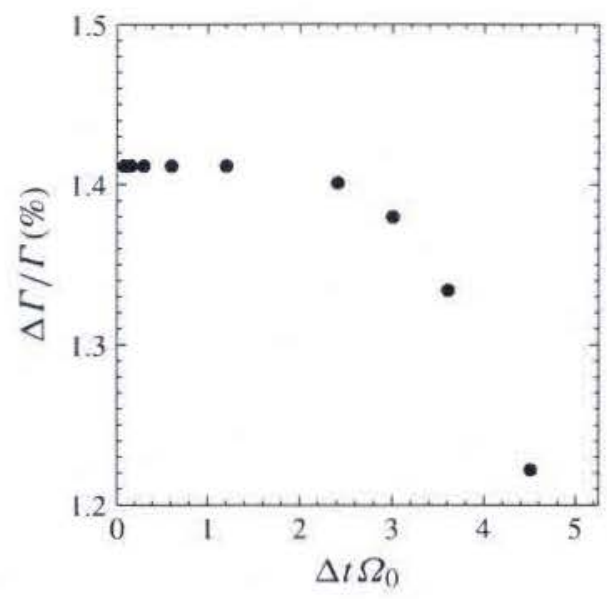

FIGURE 21. The sensitivity of the simulation to the numerical parameters. Comparison of the maximum perturbation size accepted by the $L / D=4$ model as a function of the size of the time step.

models consisting of as few as five contours. In our contour dynamics simulations, we demonstrated that nested regions of vorticity can be used to approximate dipolar solutions to the Euler equations with high fidelity. The contour dynamics models for dipolar vortices presented here are of great interest, given the widespread use of the Lamb dipole and Lamb-like vortices in studies of two-dimensional turbulence. In addition to the Lamb dipole, nested contours could be used to model the families of deformed vortices derived from the Lamb dipole by Boyd \& Ma (1990), Kizner \& Khvoles (2004) and Khvoles et al. (2005), which have also been used to model the dipolar vortices found in two-dimensional turbulence and geophysical flows.

In the case of axisymmetric vortex flows, we obtained nested-contour models for the vortex rings formed in starting jets. These models were constructed using contours of vorticity density from experimental data of a piston-cylinder experiment with a long stroke ratio, and were then allowed to asymptote to a steady state. From these steady states, we were able to construct a family of model vortex rings at different stroke ratios.

We considered the perturbation response of the model vortex rings to physically pertinent shape perturbations, which simulated the addition of a small amount of circulation to the rear of a vortex ring by the feeding shear layer. The results of the perturbation study suggest that there is a transition in the response of these vortices to this type of finite perturbation at a stroke ratio of approximately 3 . For stroke ratios below 3 , we found that the relative amount of circulation a model vortex ring could accept before shedding vorticity into a tail decreased with increasing stroke ratio. This suggests that the model vortex rings of increasing stroke ratio were progressively closer to the saturation size. Above a stroke ratio of 3 , however, the relative amount of circulation the vortex rings could accept in the form of these perturbations levelled off. This change in behaviour was consistent with vortex pinch-off: vortex rings of increasing stroke ratio were found to be increasingly sensitive to perturbation by addition of vorticity, until a stroke ratio of 3 . Model vortex rings with stroke ratios of 3 and 4 were found to be approximately equally sensitive to this type of perturbation, suggesting that they are quite similar in structure and perturbation response.

Several mechanisms that trigger the pinch-off of the leading vortex ring have been proposed to date, for a variety of configurations and flow conditions. Examples include 
the velocity of the vortex ring exceeding the velocity of the incoming shear layer (Mohseni, Ran \& Colonius 2000), and the instability of the trailing jet accelerating the detachment of the leading vortex ring (Zhao, Frankel \& Mongeau 2000). Several methods to delay or prevent pinch-off by manipulating these mechanisms have also been proposed and successfully demonstrated (Mohseni et al. 2000; Krueger \& Gharib 2003; Dabiri \& Gharib 2004, 2005b). These studies highlight the fact that pinch-off can occur via more than one mechanism, and that several factors affect the formation time of a vortex ring. While it has been suggested that the stability of the trailing jet bears a close relation to pinch-off (Zhao et al. 2000), the current results suggest that the stability of the leading vortex ring itself can also play a role that could be sufficient to explain the vortex dynamics.

The change in behaviour in the model vortices at a stroke ratio of approximately 3 reported here differs from the occurrence of pinch-off at a stroke ratio of 4 reported by Gharib et al. (1998) for circular starting jets, and at $\hat{T}=4.2 \pm 0.2$ for this particular data set (O'Farrell \& Dabiri 2010). However, given that the inviscid models were constructed from viscous experimental data and allowed vorticity to be shed until they reached a steady state that differed from the experimental vorticity contours and had lower total circulation, the qualitative robustness of the observed pinch-off phenomena is encouraging.

Future work should focus on improving the accuracy of the contour dynamics models, to develop increasingly realistic models for naturally occurring vortices and allow for quantitative comparison with experimental results. To this end, nested-contour models could be constructed using a larger class of experimental data sets, as well as for numerical data, which have the advantage of providing smoother vorticity contours from which to extract models. The accuracy of the vortex models could also potentially be improved by increasing the number of nested contours of which they are comprised.

At this stage, the greatest impediments to carrying out studies with an increased number of contours or on a larger class of data sets are the expenses associated with obtaining the asymptotic shapes of the vortex rings. In the present study, the contour shapes obtained directly from the experimental data were not found to correspond to steadily translating shapes. As a result, these shapes were allowed to approach their asymptotic states by simulating them for times up to $t^{*}=60$. This necessitated relatively costly long-time simulations, as well as labour-intensive excisions of filaments. While the existence of steadily translating vortex ring solutions to the equations of motion is well established both numerically and theoretically, the present results suggest that such solutions may be obtained from steady or quasi-steady limits from experimental data. Future work in developing a numerical method of finding equilibrium shapes using nested contours similar to Norbury's scheme (Norbury 1973) could sharply reduce these costs. The work of Aref \& Vainchtein (1998) on finding asymmetric point vortex equilibria, starting from previously known symmetric equilibria, is one potential basis for finding nested-contour solutions, starting from existing single-contour solutions.

The contour dynamics method was selected because of its simplicity and low computational cost. In addition, we have found that only a small number of contours are required to reproduce the behaviour of inviscid exact solutions using this method. However, increased computational capabilities have made high-fidelity viscous direct numerical simulations feasible in two dimensions and in axisymmetric geometries. Therefore, further work employing direct numerical simulations could yield further insight into the dynamics of pinch-off. In particular, it could allow for the extraction 
of viscous steady solutions more directly from experimental data, thus enabling a more quantitative comparison of the results of a perturbation analysis with existing experimental studies.

The present results show that only instantaneous shape perturbations to the outermost contours of the model vortex rings are required to produce a change in response with increasing stroke ratio. However, vortex rings in a starting jet are subject to the continuous injection of vorticity from a feeding shear layer. Furthermore, the vorticity in the trailing jet, while not comparable with that at the centre of the vortex ring core, certainly exceeds the levels in the outermost regions of the core (those bounded by the outermost contour in our model). Hence, in addition to improving the accuracy of the approximations to the Gaussian vorticity distributions inside vortex rings, the perturbation response method could be improved by incorporating more realistic perturbations. Such a perturbation scheme would entail the continuous injection of vorticity at the rear of the vortex, and it could be applied to multiple contour levels to reflect the relative vorticity levels in the shear layer and vortex ring.

Ultimately, the aim of this line of research is the development of a perturbation response framework for the analysis of vortex pinch-off, which is tailored to biological applications where existing models for pinch-off prove difficult to apply. Therefore, future work will focus on the application of contour dynamics methods to obtain models for the vortices shed by swimming and flying animals and in cardiovascular flows, and on extending the perturbation response methods introduced here to assess the optimality of the vortices formed in biological systems.

\section{Acknowledgements}

This work was funded by an NSF Graduate Research Fellowship to C.O'F. and by Office of Naval Research awards N000140810918 and N000141010137 to J.O.D. The authors gratefully acknowledge the contributions of Professor Tony Leonard, through many helpful discussions on the axisymmetric contour dynamics formulation.

\section{REFERENCES}

Afanasyev, Y. D. 2006 Formation of vortex dipoles. Phys. Fluids 18, 037103.

Albrecht, T. R., Elcrat, A. R. \& Miller, K. G. 2011 Steady vortex dipoles with general profile functions. J. Fluid Mech. 670, 85-95.

AREF, J. \& VAINCHTEIN, D. L. 1998 Point vortices exhibit asymmetric equilibria. Nature 392, 769-770.

BENJAMIN, T. B. 1976 The alliance of practical and analytical insights into the nonlinear problems of fluid mechanics. In Applications of Methods of Functional Analysis to Problems in Mechanics (ed. P. Germain \& B. Nayroles), pp. 8-28. Springer.

BoyD, J. P. \& MA, H. 1990 Numerical study of elliptical modons using a spectral method. J. Fluid Mech. 221, 597-611.

Chaplygin, S. A. 1903 One case of vortex motion in a fluid. Trans. Phys. Sect. Imperial Moscow Soc. Friends Nat. Sci. 11 (2), 11-14.

Corcos, G. M. \& LiN, S. J. 1984 The mixing layer: deterministic models of a turbulent flow. Part 2. The origin of the three-dimensional motion. J. Fluid Mech. 139, 67-95.

Corcos, G. M., Sherman, F. S. \& Lin, S. J. 1984 The mixing layer: deterministic models of a turbulent flow. Part 1. Introduction and the two-dimensional flow. J. Fluid Mech. 139, 29-65.

Dabiri, J. O., Colin, S. P., Katija, K. \& Costello, J. H. 2010 A wake-based correlate of swimming performance and foraging behaviour in seven co-occurring jellyfish species. J. Expl Biol. 213, 1217-1225. 
DABIRI, J. O. \& GHARIB, M. 2004 Delay of vortex ring pinch-off by an imposed bulk counterflow. Phys. Fluids 16 (L), 28-30.

DABIRI, J. O. \& GHARIB, M. $2005 a$ The role of optimal vortex formation in biological fluid transport. Proc. R. Soc. B 272, 1557-1560.

DABIRI, J. O. \& GHARIB, M. $2005 b$ Starting flow through nozzles with temporally variable exit diameter. J. Fluid Mech. 538, 111-136.

DEEM, G. S. \& ZABUSKY, N. J. 1978 Vortex waves: stationary 'V states', interactions, recurrence and breaking. Phys. Rev. Lett. 40, 859-862.

Dickinson, M. H., Farley, C. T., Full, R. J., Koehl, M. A. R., Kram, R. \& Lehman, S. 2000 How animals move: an integrative view. Science 288 (5463), 100-106.

Domenichini, F. 2011 Three-dimensional impulsive vortex formation from slender orifices. J. Fluid Mech. 666, 506-520.

DRITSCHEL, D. G. $1988 a$ Contour surgery: a topological reconnection scheme for extended integrations using contour dynamics. J. Comput. Phys. 77, 240-266.

DritsChEL, D. G. $1988 b$ The repeated filamentation of two-dimensional vorticity interfaces. J. Fluid Mech. 194, 511-547.

DRITSCHEL, D. G. 1989 Contour dynamics and contour surgery: numerical algorithms for extended high-resolution modelling of vortex dynamics in two-dimensional, inviscid, incompressible flows. Comput. Phys. Rep. 10, 79-146.

FLón, J. B. \& VAN HeIJST, G. J. F. 1994 An experimental study of dipolar vortices in a stratified fluid. J. Fluid Mech. 279, 101-133.

FUKUMOTO, Y. \& KAPLANSKI, F. 2008 Global time evolution of an axisymmetric vortex ring at low Reynolds numbers. Phys. Fluids 20, 053103.

GAO, L. \& YU, S. C. M. 2010 A model for the pinch-off process of the leading vortex ring in a starting jet. J. Fluid Mech. 656, 205-222.

Gharib, M., Rambod, E., Kheradvar, A., Sahn, D. J. \& Dabiri, J. O. 2006 Optimal vortex formation as an index for cardiac health. Proc. Natl Acad. Sci. USA 103, 6305-6308.

GHARIB, M., RAMBOD, E. \& SHARIFF, K. 1998 A universal time scale for vortex ring formation. J. Fluid Mech. 360, 121-140.

Hill, M. J. M. 1894 On a spherical vortex. Phil. Trans. R. Soc. Lond. A 185, 213-245.

JACOBS, P. A. \& Pullin, D. I. 1989 Multiple-contour-dynamic simulation of eddy scales in the plane shear layer. J. Fluid Mech. 199, 89-124.

KAPLANSKI, F. B. \& RUDI, Y. A. 2005 A model for the formation of 'optimal' vortex rings taking into account viscosity. Phys. Fluids 17, 087101.

Kelvin, L. 1880 Vortex statics. Phil. Mag. 10, 97-109.

KhVoles, R., BERSON, D. \& KIZNER, Z. 2005 The structure and evolution of elliptical barotropic modons. J. Fluid Mech. 530, 1-30.

Kizner, Z. \& KHVoles, R. 2004 Two variations on the theme of Lamb-Chaplygin: supersmooth dipoles and rotating multipoles. Regular Chaotic Dyn. 4, 509-518.

KRUeger, P. S., Dabiri, J. O. \& Gharib, M. 2006 The formation number of vortex rings in uniform background coflow. J. Fluid Mech. 556, 147-166.

Krueger, P. S. \& Gharib, M. 2003 The significance of vortex ring formation to the impulse and thrust of a starting jet. Phys. Fluids 15, 1271-1281.

LAMB, H. 1895 Hydrodynamics. 2nd edn Cambridge University Press.

L.AMB, H. 1906 Hydrodynamics. 3rd edn Cambridge University Press.

LiM, T. T. \& Nickels, T. B. 1995 Vortex rings. In Fluid Vortices, Kluwer Academic Publishers.

LIN, S. J. \& CoRCos, G. M. 1984 The mixing layer: deterministic models of a turbulent flow. Part 3. The effects of plane strain on the dynamics of streamwise vortices. J. Fluid Mech. 141, 139-178.

LiNDEN, P. F. \& TURNer, J. S. 2001 The formation of 'optimal' vortex rings, and the efficiency of propulsive devices. J. Fluid Mech. 427, 61-72.

Linden, P. F. \& TURNER, J. S. 2004 'Optimal' vortex rings and aquatic propulsion mechanisms. Proc. R. Soc. B 271, 647-653.

Lugt, H. J. 1995 Vortex Flow in Nature and Technology. Krieger Publishing Company. 
Meleshko, V. V. \& VAn Heust, G. J. F. 1994 On Chaplygin's investigations of two-dimensional vortex structures in an inviscid fluid. J. Fluid Mech. 272, 157-182.

MEllander, M. V., MCWilliams, J. C. \& ZABUSKY, N. J. 1987 Axisymmetrization and vorticititygradient intensification of an isolated two-dimensional vortex through filamentation. J. Fluid Mech. 178, 137-158.

MOHSEni, K. \& GHARIB, M. 1998 A model for universal time scale of vortex ring formation. Phys. Fluids 10, 2436-2438.

Mohseni, K., RAN, H. \& ColoniUs, T. 2000 Numerical experiments on vortex ring formation. J. Fluid Mech. 430, 267-282,

NORBURY, J. 1973 A family of steady vortex rings. J. Fluid Mech. 57, 417-431.

O'FARRELL, C. \& DabiRI, J. O. 2010 A Lagrangian approach to vortex pinch-off. Chaos $20,017513$.

O'FARRELL, C. \& DABIRI, J. O. 2012 Perturbation response and pinch-off of vortex rings and dipoles. J. Fluid Mech. 704, 280-300.

Pawlak, G., Cruz, C. M., Bazan, C. M. \& Hrdy, P. G. 2007 Experimental characterization of starting jet dynamics. Fluid Dyn. Res. 39, 711-730.

Pedrizzetti, G. 2010 Vortex formation out of two-dimensional orifices, J. Fluid Mech. 655, $198-216$.

PIERREHUMBERT, R. T. 1980 A family of steady, translating vortex pairs with distributed vorticity. J. Fluid Mech. 99, 129-144.

POZRIKIDIS, C. 1986 The nonlinear instability of Hill's vortex. J. Fluid Mech. 168, 337-367.

Pullin, D. I. 1981 The nonlinear behavour of a constant vorticity layer at a wall. J. Fluid Mech. 108, $401-421$.

Pullin, D. I. \& JACOBS, P. A. 1986 Inviscid evolution of stretched vortex arrays. J. Fluid Mech. $171,377-406$.

RAYNER, J. M. V. 1979 A vortex theory of animal flight. Part 2: the forward flight of birds. J. Fluid Mech. 91, 731-763.

SharifF, K. \& Leonard, A. 1992 Vortex rings. Annu. Rev. Fluid Mech. 24, 235-279.

SHARIFF, K., LEONARD, A. \& FERZIGER, J. H. 2008 A contour dynamics algorithm for axisymmetric flow. J. Comput. Phys, 227, 9044-9062.

Shusser, M. \& GHARIB, M. 2000 Energy and velocity of a forming vortex ring. Phys. Fluids 12, 618-621.

Trieling, R., Santberg, R., van Heijst, G. J. F. \& Kizner, Z. 2010 Barotropic elliptical dipoles in a rotating fluid. Theor. Comput. Fluid Dyn. 24, 111-115.

TURner, J. S, 1960 On the intermittent release of smoke from chimneys. Mech. Engng. Sci. 2, 97-100.

VAN Geffen, J. H. G. M. \& VAN HeIst, G. J. F. 1998 Viscous evolution of 2D dipolar vortices. Fluid Dyn. Res. 22, 191-213.

WeigAnd, A. \& GHARIB, M. 1997 On the evolution of laminar vortex rings. Exp. Fluids 22, $447-457$.

YE, Q. Y. \& CHU, C. K. 1995 Unsteady evolutions of vortex rings. Phys. Fluids 7, 795-801.

ZABUSKY, N. J., HUGHeS, M. H. \& ROBERTS, K. V. 1979 Contour dynamics for the Euler equations in two dimensions, J. Comput. Phys. 30, 96-106.

ZhaO, W., Frankel, S. H. \& MongeAU, L. G. 2000 Effects of trailing jet instability on vortex ring formation. Phys. Fluids 12, 589-621. 